\title{
Pincement spectral en courbure de Ricci positive
}

\author{
Jérôme Bertrand
}

Résumé. Dans cet article, nous démontrons que sur les variétés riemanniennes de dimension $n$ vérifiant Ric $\geq(n-1) g$ et pour $k$ dans $\{1, \ldots, n+1\}$, la $k^{\mathrm{e}}$ valeur propre du laplacien est proche de $n$ si et seulement si la variété contient une partie Gromov-Hausdorff proche de la sphère $\mathbb{S}^{k-1}$. Pour $k=n+1$, nous obtenons une nouvelle preuve des résultats de Petersen et Colding qui montrent que pour de telles variétés, la $(n+1)^{\mathrm{e}}$ valeur propre est proche de $n$ si et seulement si la variété est Gromov-Hausdorff proche de la sphère de dimension $n$.

Abstract. We show that for $n$-dimensional manifolds with Ric $\geq(n-1) g$ and for $k$ in $\{1, \ldots, n+1\}$, the $k$-th eigenvalue for the Laplacian is close to $n$ if and only if the manifold contains a subset which is Gromov-Hausdorff close to the sphere $\mathbb{S}^{k-1}$. For $k=n+1$, this gives a new proof of results of Colding and Petersen which show that the $(n+1)$-th eigenvalue is close to $n$ if and only if the manifold is Gromov-Hausdorff close to the $n$-sphere.

Codes AMS (2000). 53C20, 58J50.

Mots-clés. Géométrie riemannienne, pincement, distance de Gromov-Hausdorff.

\section{Introduction}

Dans cet article, nous considérons les variétés riemanniennes connexes, compactes $(M, g)$ de dimension $n$ dont la courbure de Ricci vérifie l'inégalité Ric $\geq(n-1) g$. On note $\mathcal{M}_{\mathrm{n}}$ l'ensemble (des classes d'isométrie) de ces variétés. Sur $\mathcal{M}_{\mathrm{n}}$, la sphère canonique $\left(\mathbb{S}^{n}, g_{\text {can }}\right)$ réalise l'extrémum de plusieurs invariants riemanniens.

Théorème 1 ([15], [3], [14], [5], [16]). Tout élément $(M, g)$ de $\mathcal{M}_{\mathrm{n}}$ vérifie

$$
\operatorname{diam}(M) \leq \pi, \quad \operatorname{vol}(M) \leq \operatorname{vol}\left(\mathbb{S}^{n}\right), \quad \lambda_{1}(M) \geq n,
$$

où $\lambda_{1}(M)$ désigne la première valeur propre (non nulle) du laplacien de $(M, g)$ agissant sur les fonctions. De plus, dans chaque inégalité, l'égalité n'a lieu que si la variété $(M, g)$ est isométrique à la sphère canonique.

L'objet de cet article est de caractériser les variétés appartenant à $\mathcal{M}_{\mathrm{n}}$ dont le début du spectre est presque minimal (c'est-à-dire proche de $n$ ). De nombreux auteurs se 
sont intéressés à cette question de la presque égalité (aussi appelée pincement) pour des invariants riemanniens comme le volume ou le diamètre. Nous rappelons cidessous quelques-uns de ces résultats, qui ont plus particulièrement motivé ce travail. Le premier d'entre eux est que la première valeur propre (non nulle) du laplacien d'un élément de $\mathcal{M}_{\mathrm{n}}$ est proche de $n$ si et seulement si le diamètre de cette variété est proche de $\pi$ (la condition nécessaire est due à S.Y. Cheng [5], la condition suffisante à C. Croke [8]). Plus précisément, l'équivalence est la suivante :

Théorème 2 ([5], [8]). Pour tout réel positif $\varepsilon$, il existe un réel positif $\eta$ tel que tout élément $(M, g)$ de $\mathcal{M}_{\mathrm{n}}$ pour lequel $\operatorname{diam}(M)>\pi-\eta$ (respectivement $\lambda_{1}(M)<$ $n+\eta$ ), vérifie $\lambda_{1}(M)<n+\varepsilon$ (respectivement $\operatorname{diam}(M)>\pi-\varepsilon$ ).

Dans la suite, nous écrirons simplement « est proche de » pour ce type d'équivalence.

En 1996, T. Colding a démontré des résultats de pincement faisant intervenir la distance de Gromov-Hausdorff (nous renvoyons à [11] pour la définition de cette distance) :

Théorème 3 ([7], [6]). Pour tout élément $(M, g)$ de $\mathcal{M}_{\mathrm{n}}$, les propriétés suivantes sont équivalentes :

1) $\operatorname{vol}(M)$ est proche de $\operatorname{vol}\left(\mathbb{S}^{n}\right)$,

2) $\operatorname{rad}(M)$ est proche de $\pi$,

3) $d_{G H}\left(M, \mathbb{S}^{n}\right)$ est proche de 0 ,

où $\operatorname{rad}(M)$ est le plus petit rayon d'une boule recouvrant $M$ et $d_{G H}$ désigne la distance de Gromov-Hausdorff.

Par la suite, P. Petersen a obtenu une nouvelle condition équivalente faisant intervenir le spectre du laplacien. Rappelons que sur la sphère canonique $\mathbb{S}^{n}$, la première valeur propre non nulle $n$ est de multiplicité $n+1$.

Théorème 4 ([17]). Pour tout élément $(M, g)$ de $\mathcal{M}_{\mathrm{n}}$, les conditions suivantes sont équivalentes:

1) $\operatorname{rad}(M)$ est proche de $\pi$,

2) $\lambda_{n+1}(M)$ est proche de $n$.

Pour énoncer le résultat principal de cet article, nous avons besoin de la définition suivante.

Définition 1. Soit $(M, g)$ un élément de $\mathcal{M}_{\mathrm{n}}, k$ un entier positif et $\eta$ un nombre réel positif ou nul. La variété $(M, g)$ vérifie la propriété $P_{k}(\eta)$ s'il existe $k$ couples de points $\left(x_{1}, y_{1}\right), \ldots,\left(x_{k}, y_{k}\right)$ dans $M^{2}$, vérifiant pour tout $i$ dans $\{1, \ldots, k\}$,

$$
d\left(x_{i}, y_{i}\right)>\pi-\eta,
$$


et pour tout $i, j$ distincts dans $\{1, \ldots, k\}$,

$$
\left|d\left(x_{i}, x_{j}\right)-\frac{\pi}{2}\right|<\eta \text {. }
$$

Dans cet article, nous démontrons le

Théorème 5. Soit $k$ dans $\{1, \ldots, n+1\}$. Pour tout élément $(M, g)$ de $\mathcal{M}_{\mathrm{n}}$, les propriétés suivantes sont équivalentes :

1) $\lambda_{k}(M)$ est proche de $n$,

2) $(M, g)$ vérifie la propriété $P_{k}(\eta)$ pour $\eta$ proche de 0 ,

3) $(M, g)$ contient une partie $A_{k}$ telle que $d_{G H}\left(A_{k}, \mathbb{S}^{k-1}\right)$ est proche de 0.

De plus, si la troisième propriété est satisfaite, la partie $A_{k}$ vérifie également une propriété de "presque convexité ». Nous renvoyons à la proposition 4.5 pour plus de détails.

Lorsque $k=1$, l'énoncé du théorème 5 se ramène à celui du théorème 2 . Lorsque $k=n+1$, notre démonstration fournit en particulier une nouvelle preuve de la propriété

$$
\lambda_{n+1}(M) \text { proche de } n \text {, implique } d_{G H}\left(M, \mathbb{S}^{n}\right) \text { proche de } 0,
$$

autrement dit, on peut prendre $A_{n+1}=M$ dans l'énoncé ci-dessus.

Sous les hypothèses du théorème 4, P. Petersen montre également que l' application $\Phi=\frac{\left(f_{1}, \ldots, f_{n+1}\right)}{\sqrt{f_{1}^{2}+\cdots+f_{n+1}^{2}}}$ est une approximation de Gromov-Hausdorff de $M$ sur la sphère canonique, où les $\left(f_{i}\right)_{1 \leq i \leq n+1}$ sont les fonctions propres associées à $\left(\lambda_{i}(M)\right)_{1 \leq i \leq n+1}$ et normalisées par analogie avec le cas de la sphère. Une étape importante de la démonstration de P. Petersen de l'implication (1) est de prouver que l'application $\Phi$ est surjective. Pour cela P. Petersen montre que le degré de $\Phi$ est non nul ${ }^{1}$. Dans notre cas, il n'y a pas de raison pour que la partie $A_{k}$ soit une variété de dimension $k-1$ et donc on ne peut pas appliquer un argument de degré. Nous utilisons à la place un lemme de Toponogov $L^{2}$ initialement introduit par T. Colding dans [7] mais contrairement à $\mathrm{T}$. Colding nous ne fixons pas des conditions au bord pour l'équation différentielle sous-jacente mais des conditions initiales de Cauchy. Ceci nous permet d'obtenir une nouvelle preuve du résultat de C. Croke et même d'obtenir un lien précis entre la fonction propre et les points à distance presque $\pi$ (voir la proposition 3.2). Le contrôle de la condition initiale sur la dérivée est une conséquence d'une estimation du gradient d'une fonction propre due à $\mathrm{P}$. Li et $\mathrm{S}$. T. Yau.

Cet article est organisé de la manière suivante. Dans la deuxième section, nous donnons les estimations sur les fonctions propres qui nous seront nécessaires pour

\footnotetext{
${ }^{1}$ P. Petersen a reconnu avoir commis une erreur dans sa démonstration de la surjectivité [18], la preuve du théorème 5 donne en particulier une preuve complète de la propriété (1).
} 
démontrer le théorème 5 , nous présentons également le lemme de Toponogov $L^{2}$ (lemme 1.6) et l'utilisation que nous allons en faire. Dans la troisième section, nous démontrons qu'un élément de $\mathcal{M}_{\mathrm{n}}$ vérifiant la propriété $P_{k}(\eta)$ pour $\eta$ petit a nécessairement $k$ valeurs propres proches de $n$. La quatrième section est consacrée à la réciproque. Dans la dernière partie nous montrons que la condition 1) implique la condition 3 ), ce qui termine la preuve du théorème 5 puisque la condition 3 ) implique clairement la condition 2).

\section{Résultats préliminaires}

Soit $p \geq 1$ un nombre réel et $h$ appartenant à $L^{p}(M)$. On note

$$
\|h\|_{L^{p}}=\left(\frac{1}{\operatorname{vol} M} \int_{M} h^{p} d x\right)^{\frac{1}{p}} .
$$

On utilisera la définition usuelle pour la norme $L^{\infty}$.

On notera $\tau(\varepsilon), r(\varepsilon), \eta(\varepsilon)$, etc ... de manière générique, toute quantité positive ne dépendant que de $\varepsilon$ et de la dimension $n$ de la variété, dont la limite quand $\varepsilon$ tend vers 0 est 0 .

Enfin, toute fonction propre $f$ de valeur propre proche de $n$ sera normalisée par analogie avec le cas de la sphère, par

$$
\frac{1}{\operatorname{vol} M} \int_{M} f^{2}=\frac{1}{n+1}
$$

Les fonctions propres de $\left(\mathbb{S}^{n}, g_{\text {can }}\right)$ associées à la valeur propre $n$ sont les fonctions $\cos d_{x}$, avec $x$ appartenant à $\mathbb{S}^{n}$ et $d_{x}$ la fonction distance au point $x$. En particulier, elles vérifient

$$
\begin{aligned}
& \cos ^{2} d_{x}+\left|\nabla \cos d_{x}\right|^{2}=1, \\
& \frac{1}{\operatorname{vol}\left(\mathbb{S}^{n}\right)} \int_{\mathbb{S}^{n}} \cos ^{2} d_{x}=\frac{1}{n+1} .
\end{aligned}
$$

Sur une variété de $\mathcal{M}_{\mathrm{n}}$ admettant des valeurs propres proches de $n$, on a l'estimation suivante due à $\mathrm{P}$. $\mathrm{Li}$ :

Proposition 1.1 ([13]). Soit $\left(M^{n}, g\right)$ une variété riemannienne compacte de dimension $n$ dont la courbure de Ricci vérifie Ric $\geq(n-1) g$. Soit $\bar{f}$ une combinaison linéaire de fonctions propres du laplacien $\operatorname{sur}(M, g)$

$$
\bar{f}=\sum_{i=1}^{k} a_{i} f_{i},
$$


avec $k$ un entier non nul et $\Delta f_{i}=\lambda_{i} f_{i}$ pour tout $i$ dans $\{1, \ldots, k\}$.

Supposons que pour tout $i$ dans $\{1, \ldots, k\}$,

$$
\lambda_{i} \leq n+\varepsilon
$$

avec $\varepsilon>0$, alors

$$
\left\|\bar{f}^{2}+|d \bar{f}|^{2}\right\|_{L^{\infty}} \leq(1+\tau(\varepsilon))(n+\varepsilon+1)\|\bar{f}\|_{L^{2}}^{2},
$$

où $\tau(\varepsilon)$ est une fonction croissante ne dépendant que de $n$ et telle que $\lim _{\varepsilon \rightarrow 0} \tau(\varepsilon)=0$.

Remarque 1.2. En particulier si $f$ est une fonction propre de valeur propre non nulle $\lambda \leq n+\varepsilon$ et si $\frac{1}{\operatorname{vol} M} \int_{\mathrm{M}} f^{2}=\frac{1}{n+1}$, on obtient pour tout $x$ dans $M$,

$$
f^{2}(x)+|d f|^{2}(x) \leq 1+\tau(\varepsilon),
$$

donc comme $\frac{1}{\text { vol M }} \int_{\mathrm{M}} f^{2}+|d f|^{2}=\frac{\lambda+1}{n+1}$, on en déduit que (3) est « stable » pour la norme $L^{1}$ :

$$
\left.\frac{1}{\operatorname{volM}} \int_{M}\left|f^{2}+\right| d f\right|^{2}-1 \mid \leq \tau(\varepsilon) .
$$

D'autre part, si l'on suppose $\varepsilon<1$ alors il existe une constante $C(n)$ ne dépendant que de la dimension $n$ de $M$, telle que

$$
\left\|f^{2}+|\nabla f|^{2}\right\|_{L^{\infty}} \leq C(n),
$$

nous utiliserons implicitement cette propriété par la suite.

La preuve de ce type d'inégalité est essentiellement classique, elle repose sur une inégalité de Sobolev et le procédé d'itération de Moser. Nous renvoyons à [10] et à $[2$, lemme 1.4] pour une démonstration dans ce cas particulier. Une conséquence de cette proposition est qu'un élément de $\mathcal{M}_{\mathrm{n}}$ admet au plus $n+1$ valeurs propres proches de $n$.

Corollaire 1.3 ([10]). Il existe une constante $C(n)>0$ telle que pour tout élément $(M, g)$ de $\mathcal{M}_{\mathrm{n}}$,

$$
\lambda_{n+2}(M) \geq n+C(n) .
$$

Une autre propriété caractéristique des fonctions $\cos d_{x}$ sur la sphère $\left(\mathbb{S}^{n}, g_{\text {can }}\right)$ est qu'elles sont solutions de l'équation

$$
\text { Hess } f+f g_{\text {can }}=0 .
$$

Un résultat de M. Obata [16] montre que ce sont les seules solutions parmi les fonctions régulières définies sur un élément $(M, g)$ de $\mathcal{M}_{\mathrm{n}}$. Cependant, à l'aide de la formule de Bochner, on montre (voir par exemple [7], page 178) la 
Proposition 1.4. Il existe une constante $C(n)$ telle que tout élément $(M, g)$ de $\mathcal{M}_{\mathrm{n}}$ pour lequel $n \leq \lambda_{1}(M) \leq n+\varepsilon$, vérifie l'inégalité

$$
\| \text { Hess } f+f g\left\|_{L^{2}} \leq C(n) \varepsilon^{\frac{1}{2}}\right\| f \|_{L^{2}},
$$

où $f$ est une fonction propre associée à $\lambda_{1}(M)$.

Remarque 1.5. Il existe des éléments de $\mathcal{M}_{\mathrm{n}}$ non homéomorphes à la sphère dont la première valeur propre est arbitrairement proche de $n$ (voir par exemple [1]). Un résultat de $\mathrm{S}$. Gallot [9, lemme 3.1] implique alors qu'on ne peut espérer obtenir une estimation similaire de Hess $f+f g$ en norme $L^{\infty}$.

Le lemme suivant permet de déduire des informations géométriques de cette inégalité sur le hessien.

Lemme 1.6 ([4, Theorem 2.11]). Soit $(M, g)$ un élément de $\mathcal{M}_{\mathrm{n}}$. Il existe des constantes, ne dépendant que de $n$, notées $C(n)$ et $\tilde{C}(n)$, telles que pour $x_{1}$ et $x_{2}$ appartenant à $M, r_{1}, r_{2}$ des réels positifs (on note $B_{i}=B\left(x_{i}, r_{i}\right)$ ) et pour toute fonction continue $h$ sur $M$, on a

$$
\begin{aligned}
& \frac{1}{\operatorname{vol}\left(B_{1} \times B_{2}\right)} \int_{B_{1} \times B_{2}}\left(\oint_{\gamma_{x y}} h^{2}\right) d x d y \\
& \leq C(n)\left(\frac{1}{\operatorname{vol} B_{1}}+\frac{1}{\operatorname{vol} B_{2}}\right) \int_{M} h^{2}(x) d x .
\end{aligned}
$$

On obtient, en particulier, pour $r_{1}=r_{2}=r$

$$
\frac{1}{\operatorname{vol}\left(B_{1} \times B_{2}\right)} \int_{B_{1} \times B_{2}}\left(\oint_{\gamma_{x y}} h^{2}\right) d x d y \leq \frac{\tilde{C}(n)}{V(r)} \frac{1}{\operatorname{vol}(M)} \int_{M} h^{2}(x) d x
$$

Remarque 1.7. La notation $V(r)$ désigne le volume d'une boule géodésique de $\left(\mathbb{S}^{n}, g_{\text {can }}\right)$ de rayon $r$. La notation $B_{1} \times B_{2}$ désigne en réalité le sous-ensemble de mesure pleine de ce produit, constitué par les couples admettant une unique géodésique minimisante les reliant (notée $\gamma_{x y}$ ). La seconde inégalité se déduit de la première en utilisant le théorème de Bishop-Gromov (nous renvoyons à [11] pour un énoncé).

En appliquant ce lemme à la fonction $\mid$ Hess $f+f g \mid$ où $f$ est la fonction propre associée à $\lambda_{1}(M)$ normalisée par (2), dans le cas où $r_{1}=r_{2}=r$ et en remarquant que pour une géodésique paramétrée par longueur d'arc

$$
\left|\left(f \circ \gamma_{x y}\right)^{\prime \prime}(t)+\left(f \circ \gamma_{x y}\right)(t)\right|^{2} \leq|\operatorname{Hess} f+f g|^{2},
$$


on déduit de la proposition 1.4

$$
\frac{1}{\operatorname{vol}\left(B_{1} \times B_{2}\right)} \int_{B_{1} \times B_{2}} \int_{0}^{d(x, y)}\left|\left(f \circ \gamma_{x y}\right)^{\prime \prime}(t)+\left(f \circ \gamma_{x y}\right)(t)\right|^{2} d t d x d y \leq C(n) \frac{\varepsilon}{V(r)} .
$$

Par conséquent, pour des rayons $r(\varepsilon)$ convenables (i.e. tels que $\frac{\varepsilon}{V(r(\varepsilon))}$ soit petit), l'inégalité de Byenaimé-Tchebitchev implique l'existence de points $x, y$ pour lesquels $f \circ \gamma_{x y}$ vérifie presque la même équation différentielle que dans le cas de la sphère canonique. On peut ensuite par des méthodes classiques comparer $f \circ \gamma_{x y}$ à une solution correspondante sur la sphère en fixant des conditions au bord (comme l'a fait T. Colding dans [7]) à l'aide du lemme suivant.

Lemme 1.8. Soit v $(t)$ et $Z(t)$ deuxfonctions définies sur $[0, l]$ avec $l<\pi$. On suppose que $\int_{0}^{l} Z^{2}(t) d t<\varepsilon^{2}$ et que $v$ est solution de $v^{\prime \prime}+v=Z$ avec $|v(0)-a|<\eta$ et $|v(l)-b|<\eta$. Il existe une constante positive $C$ telle que pour tout $t$ dans $[0, l]$,

et

$$
\left|v(t)-\tilde{u}_{a, b}(t)\right|<\frac{C}{\sin (l)}(\varepsilon+\eta)
$$

$$
\left|v^{\prime}(t)-\tilde{u}_{a, b}^{\prime}(t)\right|<\frac{C}{\sin (l)}(\varepsilon+\eta),
$$

où $\tilde{u}_{a, b}$ est la solution de $u^{\prime \prime}+u=0$ sur $[0, l]$ vérifiant les conditions initiales $u(0)=a$ et $u(l)=b$.

On peut également fixer des conditions de Cauchy.

Lemme 1.9. Soit $v(t)$ et $Z(t)$ deux fonctions définies sur $[0, l]$ avec $l \leq \pi$. On suppose que $\int_{0}^{l} Z^{2}(t) d t<\varepsilon^{2}$ et que $v$ est solution de $v^{\prime \prime}+v=Z$ avec $|v(0)-a|<\eta$ et $\left|v^{\prime}(0)-b\right|<\eta$. Il existe une constante positive $C$ telle que pour tout $t$ dans $[0, l]$,

et

$$
\left|v(t)-u_{a, b}(t)\right|<C(\varepsilon+\eta)
$$

$$
\left|v^{\prime}(t)-u_{a, b}^{\prime}(t)\right|<C(\varepsilon+\eta),
$$

où $u_{a, b}$ est la solution de $u^{\prime \prime}+u=0$ sur $[0, l]$ vérifiant les conditions initiales $u(0)=a$ et $u^{\prime}(0)=b$.

Pour contrôler les conditions initiales de l'équation différentielle dans le lemme 1.9, nous aurons besoin d'une estimation due à P. Li et S. T. Yau ([13], voir également [19], page 108) qui prouve que la norme du gradient d'une fonction propre sur un élément de $\mathcal{M}_{\mathrm{n}}$, reste petite au voisinage des points réalisant les extréma de la fonction propre. Ce résultat ne peut découler directement d'une estimation sur le hessien de la fonction propre car on constate, en considérant des sphères rondes de rayon arbitrairement petit, que la norme $L^{\infty}$ du hessien d'une fonction propre de norme 1 , tend vers l'infini. 
Proposition 1.10 ([13]). Soit $(M, g)$ un élément de $\mathcal{M}_{\mathrm{n}}$ et $f$ une fonction propre de valeur propre non nulle $\lambda$. Sous ces hypothèses, on a pour tout $x$ dans $M$, l'estimation

$$
|\nabla f|^{2}(x) \leq \frac{2 \lambda \sup _{M} f}{\sup _{M} f-\inf _{M} f}\left(\sup _{M} f-f(x)\right)\left(f(x)-\inf _{M} f\right) .
$$

\section{Variétés vérifiant la propriété $P_{k}(\eta)$}

L'objet de cette partie est de démontrer le

Théorème 2.1. Soit $k$ dans $\{2, \ldots, n+1\}$. Il existe une fonction $\tau(\eta)$ telle que, pour tout élément $(M, g)$ de $\mathcal{M}_{\mathrm{n}}$ vérifiant la propriété $P_{k}(\eta)$, on a l'estimation

$$
\lambda_{k}(M) \leq n+\tau(\eta) \text {. }
$$

La démonstration du théorème 2.1 repose en partie sur l'utilisation de fonctions $\cos d_{p}$ pour $p$ appartenant à $M$ et admettant un "presque antipode » (c'est-à-dire, $p$ est tel que $\sup _{x \in M} d(p, x)$ est proche de $\left.\pi\right)$. Nous étudions de telles fonctions dans le prochain paragraphe.

2.1. Propriétés des fonctions $\cos d_{p}$. Sur la sphère canonique, toute fonction (propre) $\cos d_{p}$ est une combinaison linéaire d'une base de fonctions propres associées à la valeur propre $n$. Précisément, si $\left(x_{i}\right)_{1 \leq i \leq n+1}$ est une base orthonormée de l'espace euclidien $\mathbb{R}^{n+1}$ alors pour tout élément $p$ de $\mathbb{S}^{n}$

$$
\cos d_{p}=\sum_{i=1}^{n+1} \cos d\left(p, x_{i}\right) \cos d_{x_{i}} .
$$

En particulier si $p$ appartient à $\mathbb{S}^{k-1}$ (en identifiant $\mathbb{S}^{k-1}$ à la partie de $\mathbb{S}^{n}$ dont les $n-k+1$ dernières coordonnées sont nulles), seuls les $p$ premiers termes de la somme ci-dessus sont non tous nuls. Nous allons montrer que la propriété (7) sur les fonctions $\cos d_{p}$ est «stable» pour les points $p$ admettant un presque antipode. Ce résultat améliore un lemme démontré par P. Petersen [17, lemme 4.3].

Lemme 2.2. Il existe une fonction $\tau(t)$ tendant vers 0 avec $t$, telle que pour tout nombre réel positif $\varepsilon, \eta$ vérifiant $\eta<\varepsilon$ et pour tout élément $(M, g)$ de $\mathcal{M}_{\mathrm{n}}$ contenant deux points $p, q$ vérifiant $d(p, q)>\pi-\eta$, on a

$$
\left\|\cos d_{p}-\sum_{i=1}^{k} a_{i}(p) f_{i}\right\|_{L^{\infty}} \leq \tau\left(\frac{\eta}{\varepsilon}\right),
$$


où $k=\max \left\{i ; \lambda_{i}(M) \leq n+\varepsilon\right\}$ est un entier non nul et où les $a_{i}(p)$ sont les coefficients de Fourier de la fonction $\cos d_{p}$ par rapport à une base orthogonale $\left(f_{i}\right)_{i \geq 0}$ de fonctions propres normalisées par (2), c'est-à-dire $a_{i}(p)=\frac{n+1}{\operatorname{vol} M} \int_{M} \cos d_{p} f_{i}$. De plus, les coefficients $a_{i}(p)$ vérifient pour $\varepsilon$ assez petit

$$
\left|\sum_{i=1}^{k} a_{i}^{2}(p)-1\right| \leq C(n) \frac{\eta}{\varepsilon} .
$$

Preuve. Soit $p$ comme dans l'énoncé. Dans la suite, on note $\left(a_{i}\right)_{i \in \mathbb{N}}$ les coefficients de Fourier de $\cos d_{p}$. Une conséquence de la formule de la coaire et du théorème de Bishop-Gromov est le

Lemme 2.3. Il existe une constante $C(n)$ telle que pour tout élément $(M, g)$ de $\mathcal{M}_{\mathrm{n}}$, admettant deux points $p$ et $q$ vérifiant $d(p, q)>\pi-\eta$ et pour toute fonction $u:[0, \pi] \rightarrow \mathbb{R}$ de classe $C^{1}$, on a

$$
\left|\frac{1}{\operatorname{vol} M} \int_{\mathrm{M}} u \circ d_{p} d v-\frac{1}{\operatorname{vol} \mathbb{S}^{\mathrm{n}}} \int_{\mathbb{S}^{n}} u \circ d_{\mathbb{S}^{n}}(\bar{p}, \cdot) d x\right| \leq C(n) \eta \int_{0}^{\pi}\left|u^{\prime}(r)\right| d r .
$$

Remarque 2.4. On obtient en particulier que $\frac{1}{\operatorname{vol}(M)} \int_{M} \cos ^{2} d_{p}$ est proche de $\frac{1}{n+1}$ si $p$ admet un presque antipode.

Nous renvoyons à [2] pour une démonstration. En appliquant le lemme 2.3 à $u=\cos ^{2}, u=\sin ^{2}$ et $u=\cos$ puis en remarquant que les expressions ci-dessous sont nulles dans le cas de la sphère canonique, on obtient

$$
\begin{gathered}
\left|\left\|\nabla \cos d_{p}\right\|_{L^{2}}^{2}-n\left\|\cos d_{p}\right\|_{L^{2}}^{2}\right| \leq C(n) \eta, \\
\left|a_{0}\right|=\left|\frac{\sqrt{1+n}}{\operatorname{vol}(M)} \int_{M} \cos d_{p}\right| \leq C(n) \eta .
\end{gathered}
$$

Par conséquent, les coefficients de Fourier de la fonction $\cos d_{p}$ vérifient

$$
\sum_{i=1}^{+\infty} \lambda_{i} a_{i}^{2}\left\|f_{i}\right\|_{L^{2}}^{2} \leq n \sum_{i=1}^{+\infty} a_{i}^{2}\left\|f_{i}\right\|_{L^{2}}^{2}+C^{\prime}(n) \eta .
$$

C'est-à-dire, d'après la normalisation (2) des fonctions propres

$$
\sum_{i=1}^{+\infty} \lambda_{i} a_{i}^{2} \leq n \sum_{i=1}^{+\infty} a_{i}^{2}+C^{\prime \prime}(n) \eta
$$


Comme $\lambda_{1}(M) \geq n$, on peut négliger les $k$ premiers termes, on obtient

$$
\sum_{i=k+1}^{+\infty}\left(\lambda_{i}-n\right) a_{i}^{2} \leq C^{\prime \prime}(n) \eta
$$

On en déduit par définition de $k$,

$$
\left\|\cos d_{p}-\sum_{i=1}^{k} a_{i} f_{i}\right\|_{L^{2}}^{2} \leq C^{\prime \prime}(n) \frac{\eta}{\varepsilon}
$$

et comme $\frac{1}{\text { vol M }} \int_{\mathrm{M}} \cos ^{2} d_{p}$ est proche de $\frac{1}{n+1}$, la formule de Parseval donne

$$
\left|\sum_{i=1}^{k} a_{i}^{2}-1\right| \leq \bar{C}(n) \frac{\eta}{\varepsilon}
$$

L'inégalité précédente montre en particulier que les coefficients $\left(a_{i}\right)_{1 \leq i \leq k}$ sont bornés. On déduit alors de l'hypothèse sur $\lambda_{k}(M)$ et de la proposition 1.1, l'existence d'une constante $D(n)$ telle que la fonction $h=\cos d_{p}-\sum_{i=1}^{k} a_{i} f_{i}$ vérifie

$$
\|h\|_{L^{\infty}} \leq D(n)
$$

et

$$
\|d h\|_{L^{\infty}} \leq D(n) .
$$

Or par l'inégalité de Cauchy-Schwartz, on déduit de (9)

$$
\|h\|_{L^{1}} \leq\left(C^{\prime \prime}(n) \frac{\eta}{\varepsilon}\right)^{\frac{1}{2}}
$$

Soit $x_{0}$ tel que $\left|h\left(x_{0}\right)\right|=\|h\|_{L^{\infty}}$ et $r$ un réel positif; le théorème des accroissements finis donne

$$
\left(C^{\prime \prime}(n) \frac{\eta}{\varepsilon}\right)^{\frac{1}{2}} \operatorname{vol}(M) \geq \int_{B\left(x_{0}, r\right)}|h(x)| d x \geq\left(\|h\|_{L^{\infty}}-D(n) r\right) \operatorname{vol}\left(B\left(x_{0}, r\right)\right) .
$$

En appliquant le théorème de Bishop-Gromov, on en déduit

$$
\left(C^{\prime \prime}(n) \frac{\eta}{\varepsilon}\right)^{\frac{1}{2}} \geq \frac{V(r)}{V(\pi)}\left(\|h\|_{L^{\infty}}-D(n) r\right) .
$$

D'où le résultat en choisissant $r=r\left(\frac{\eta}{\varepsilon}\right)$ convenable. Ce qui achève la preuve du lemme 2.2. 
2.2. Démonstration du théorème 2.1. Soit $(M, g)$ un élément de $\mathcal{M}_{\mathrm{n}}$ vérifiant la propriété $P_{k}(\eta)$, en particulier $(M, g)$ vérifie

$$
\operatorname{diam}(M) \geq \pi-\eta \text {. }
$$

Sous ces hypothèses, le résultat de S. Y. Cheng cité dans l'introduction montre que $\lambda_{1}(M)$ est proche de $n$. On déduit du lemme 2.3 une estimation explicite.

Lemme 2.5. Il existe une constante $C(n)$ telle que tout élément $(M, g)$ de $\mathcal{M}_{\mathrm{n}}$ pour lequel diam $(M)>\pi-\eta$, vérifie l'inégalité

$$
\lambda_{1}(M) \leq n+C(n) \eta .
$$

Notons

$$
\varepsilon=\sqrt{\eta} \quad \text { et } \quad k_{\varepsilon}=\max \left\{k \in \mathbb{N} ; \lambda_{k}(M) \leq n+\varepsilon\right\} .
$$

Par le lemme 2.5, pour $\eta$ assez petit, $k_{\varepsilon}$ est supérieur ou égal à 1 . Notons $\left(f_{i}\right)_{1 \leq i \leq k_{\varepsilon}}$ une famille orthogonale de fonctions propres associées à $\lambda_{i}(M)$ et normalisées par $\frac{1}{\operatorname{volM}} \int_{\mathrm{M}} f_{i}^{2}=\frac{1}{n+1}$. D'autre part, notons

$$
F=\operatorname{Vect}_{L^{2}(M)}\left\{f_{1}, \ldots, f_{k_{\varepsilon}}\right\}
$$

et $P_{F}$ la projection orthogonale de $L^{2}(M)$ sur $F$. Par hypothèse sur $(M, g)$, il existe $\left(x_{1}, y_{1}\right), \ldots,\left(x_{k}, y_{k}\right)$ appartenant à $M^{2}$ tels que pour tout $i, j$ distincts dans $\{1, \ldots, k\}$,

$$
\left|d\left(x_{i}, x_{j}\right)-\frac{\pi}{2}\right| \leq \eta
$$

et pour tout $i$ dans $\{1, \ldots, k\}$,

$$
d\left(x_{i}, y_{i}\right) \geq \pi-\eta .
$$

Par conséquent, d'après le lemme 2.2 appliqué avec $\varepsilon=\sqrt{\eta}$ et $\eta$, il existe une fonction $\tau(\eta)$ telle que, pour tout $i$ dans $\{1, \ldots, k\}$,

$$
\left\|\cos d_{x_{i}}-P_{F}\left(\cos d_{x_{i}}\right)\right\|_{L^{\infty}} \leq \tau(\eta)
$$

où $P_{F}\left(\cos d_{x_{i}}\right)=\sum_{j=1}^{k_{\varepsilon}} a_{j}\left(x_{i}\right) f_{j}$ vérifie pour tout $i$

$$
\left|\sum_{j=1}^{k_{\varepsilon}} a_{j}^{2}\left(x_{i}\right)-1\right| \leq \tau(\eta) .
$$

En particulier, pour $\eta$ assez petit et pour tout $i, P_{F}\left(\cos d_{x_{i}}\right)$ n'est pas identiquement nul. Par conséquent si $k_{\varepsilon}<k$ alors la famille $\left(P_{F}\left(\cos \left(d_{x_{i}}\right)\right)_{i=1}^{k}\right.$ est liée. Notons $\left(b_{i}\right)_{i \in\{1, \ldots, k\}}$ avec $\sum_{i=1}^{k} b_{i}^{2}=1$, des coefficients tels que

$$
\sum_{i=1}^{k} b_{i} P_{F}\left(\cos d_{x_{i}}\right)=0 \text {. }
$$


Alors (11) implique

$$
\left\|\sum_{i=1}^{k} b_{i} \cos d_{x_{i}}-\sum_{i=1}^{k} b_{i} P_{F}\left(\cos d_{x_{i}}\right)\right\|_{L^{\infty}} \leq k^{\frac{1}{2}} \tau(\eta) .
$$

C'est-à-dire,

$$
\left\|\sum_{i=1}^{k} b_{i} \cos d_{x_{i}}\right\|_{L^{\infty}} \leq k^{\frac{1}{2}} \tau(\eta) .
$$

Comme $\sum_{i=1}^{k} b_{i}^{2}=1$, l'un des coefficients $b_{i_{0}}$ vérifie $\left|b_{i_{0}}\right| \geq \frac{1}{\sqrt{k}}$. Or l'estimation (13) appliquée au point $x=x_{i_{0}}$ et l'hypothèse (10) implique

$$
\left|b_{i_{0}}\right| \leq \tau(\eta)
$$

ce qui est absurde pour $\eta$ assez petit et donc $k_{\varepsilon} \geq k$, ce qui termine la preuve du théorème 2.1 .

\section{Valeurs propres proches de $n$}

L'objet de cette partie est de démontrer la réciproque du théorème 2.1.

Théorème 3.1. Il existe une fonction $\tau(\varepsilon)$ telle que tout élément $(M, g)$ de $\mathcal{M}_{\mathrm{n}}$ pour lequel $\lambda_{k}(M) \leq n+\varepsilon$, vérifie la propriété $P_{k}(\tau(\varepsilon))$.

Ce résultat découle d'une « réciproque » du lemme 2.2.

Proposition 3.2. Il existe des fonctions $\tau(\varepsilon)$ et $\psi(\varepsilon)$ telles que, pour tout élément $(M, g)$ de $\mathcal{M}_{\mathrm{n}}$ et toute fonction propre $f$ sur $M$ de valeur propre non nulle $\lambda \leq n+\varepsilon$, normalisée par $\frac{1}{\mathrm{vol} M} \int_{\mathrm{M}} f^{2}=\frac{1}{n+1}$, on a l'estimation

$$
\left\|\cos d_{x}-f\right\|_{L^{\infty}} \leq \tau(\varepsilon)
$$

avec $x$ dans $M$ tel que $f(x)=\sup _{M} f$. De plus, si y dans $M$ vérifie $f(y)=\inf _{M} f$ alors

$$
d(x, y)>\pi-\psi(\varepsilon) .
$$

Nous démontrons cette proposition dans le prochain paragraphe. 
3.1. Fonctions propres associées à une «petite» valeur propre. La preuve de la proposition 3.2 est une conséquence du lemme 1.6. La première étape consiste à estimer la borne supérieure d'une telle fonction propre.

Lemme 3.3. Il existe une fonction $\tau(\varepsilon)$ ne dépendant que de $n$, telle que pour tout élément $(M, g)$ de $\mathcal{M}_{\mathrm{n}}$ et toute fonction propre $f$ sur $M$ de valeur propre non nulle $\lambda \leq n+\varepsilon$, normalisée par $\frac{1}{\mathrm{vol} M} \int_{\mathrm{M}} f^{2}=\frac{1}{n+1}$, on a l'estimation

$$
\left|\sup _{M} f-1\right| \leq \tau(\varepsilon) .
$$

Preuve. Par la minoration de Lichnérowicz de la première valeur propre non nulle, $\lambda$ vérifie

$$
n \leq \lambda \leq n+\varepsilon .
$$

Par choix de la normalisation de $f$, on a

$$
1 \leq \frac{1}{\operatorname{vol} M} \int_{M} f^{2}+|d f|^{2} \leq 1+\frac{\varepsilon}{n+1} .
$$

Donc d'après la proposition 1.1, il existe une fonction $\tau(\varepsilon)$ telle que $f$ vérifie pour tout $x$ dans $M$

$$
f^{2}(x)+|d f|^{2}(x) \leq 1+\tau(\varepsilon) .
$$

Ainsi $f^{2}+|d f|^{2}$ est majorée par une quantité environ égale à sa moyenne, par conséquent $f^{2}+|d f|^{2}$ est $L^{1}$-proche de sa moyenne :

$$
\left.\frac{1}{\operatorname{vol} M} \int_{M}\left|1-f^{2}(x)-\right| d f\right|^{2}(x) \mid \leq \tau(\varepsilon) .
$$

Soit $x$ vérifiant $f(x)=\sup _{M} f$. Un corollaire du théorème de Bishop-Gromov [11, remarque 2.8] implique l'existence de $R(\varepsilon), \tau^{\prime}(\varepsilon)$ ne dépendant que de $n$ et de $\tilde{x}$ vérifiant $d(x, \tilde{x}) \leq R(\varepsilon)$, tels que

$$
\left.\left|f^{2}(\tilde{x})+\right| d f\right|^{2}(\tilde{x})-1 \mid \leq \tau^{\prime}(\varepsilon) .
$$

D'après la proposition 1.10 ,

$$
|d f|^{2}(\tilde{x}) \leq \tau(\varepsilon)
$$

d'où

$$
|f(\tilde{x})-1| \leq \tau(\varepsilon),
$$

ce qui permet de conclure pour $f(x)$ puisque le gradient de $f$ est borné.

Preuve de la proposition 3.2. De l'hypothèse sur la valeur propre, on déduit (proposition 1.4)

$$
\| \text { Hess } f+f g \|_{L^{2}} \leq \tau(\varepsilon) .
$$


Fixons $x$ comme dans l'énoncé et soit $u$ dans $M$ quelconque. En appliquant le lemme 1.6 aux boules $B(x, r(\varepsilon))$ et $B(u, r(\varepsilon))$ avec $r(\varepsilon)$ convenable, on obtient l'existence d'une fonction $\tau(\varepsilon)$ telle que pour tout $u$ dans $M$, il existe $\tilde{u}, \tilde{x}$ dans $M$ tels que :

- il existe une unique géodésique minimisante $\gamma$ reliant $\tilde{x}$ à $\tilde{u}$,

$-d(u, \tilde{u}) \leq r(\varepsilon), d(x, \tilde{x}) \leq r(\varepsilon)$ et

$$
\int_{0}^{d(\tilde{x}, \tilde{u})}\left|(f \circ \gamma)^{\prime \prime}(t)+(f \circ \gamma)(t)\right|^{2} d t \leq \tau(\varepsilon) .
$$

Nous allons maintenant estimer les conditions initiales $f \circ \gamma(0)$ et $(f \circ \gamma)^{\prime}(0)$ afin d'appliquer le lemme 1.9. Par le lemme 3.3, on a

$$
\left|\sup _{M} f-1\right| \leq \tau_{2}(\varepsilon) .
$$

Par conséquent, comme $\tilde{x}$ est proche de $x$ et que le gradient de $f$ est borné, on en déduit l'existence d'une fonction $\tau_{3}(\varepsilon)$ telle que

$$
|(f \circ \gamma)(0)-1| \leq \tau_{3}(\varepsilon) .
$$

D'autre part, par la proposition 1.10, il existe une fonction $\tau_{4}(\varepsilon)$ telle que $|\nabla f|(\tilde{x}) \leq$ $\tau_{4}(\varepsilon)$ d'où

$$
\left|(f \circ \gamma)^{\prime}(0)\right| \leq \tau_{4}(\varepsilon) .
$$

Grâce à (15), (16) et (17), le lemme 1.9 appliqué à $f \circ \gamma$ et cos, implique l'existence d'une fonction $\tau_{5}(\varepsilon)$ telle que pour tout $t$ dans $[0, d(\tilde{x}, \tilde{u})]$,

$$
\begin{aligned}
& |(f \circ \gamma)(t)-\cos (t)| \leq \tau_{5}(\varepsilon) . \\
& \left|(f \circ \gamma)^{\prime}(t)+\sin (t)\right| \leq \tau_{5}(\varepsilon) .
\end{aligned}
$$

En particulier,

$$
|f(\tilde{u})-\cos (d(\tilde{x}, \tilde{u}))| \leq \tau_{5}(\varepsilon) .
$$

Donc par construction de $\tilde{x}$ et $\tilde{u}$ et comme le gradient de $f$ est borné, on en déduit l'existence d'une fonction $\tau_{6}(\varepsilon)$ telle que

$$
\|f-\cos d(x, \cdot)\|_{L^{\infty}} \leq \tau_{6}(\varepsilon) .
$$

Montrons maintenant la deuxième partie de l'énoncé. Soit $y$ vérifiant $f(y)=$ $\inf _{M} f$ et soit $\tilde{x}$ et $\tilde{y}$ comme ci-dessus.

D'après (19)

$$
\left|(f \circ \gamma)^{\prime}(d(\tilde{x}, \tilde{y}))+\sin (d(\tilde{x}, \tilde{y}))\right| \leq \tau_{5}(\varepsilon) .
$$


Comme $\tilde{y}$ est proche de $y$ qui est un point réalisant le minimum de $f$, on déduit de la proposition 1.10 apliquée à $\tilde{y}$ et de (20)

$$
|\sin (d(\tilde{x}, \tilde{y}))| \leq \tau_{7}(\varepsilon) .
$$

La borne sur le gradient de $f$ et le lemme 3.3 excluent l'hypothèse que $d(\tilde{x}, \tilde{y})$ soit proche de 0 donc les points $x$ et $y$ sont nécessairement à distance presque $\pi$.

3.2. Démonstration du théorème 3.1. Pour cela, on prouve un résultat un peu plus précis :

Proposition 3.4. Soit $k$ dans $\{2, \ldots, n+1\}$. Il existe une fonction $\tau(\varepsilon)$ telle que pour tout élément $(M, g)$ de $\mathcal{M}_{\mathrm{n}}$ vérifiant $\lambda_{k}(M) \leq n+\varepsilon$, il existe $\left(x_{1}, y_{1}\right), \ldots,\left(x_{k}, y_{k}\right)$ dans $M^{2}$ tels que, pour tout $i$ dans $\{1, \ldots, k\}$,

$$
\left|d\left(x_{i}, y_{i}\right)-\pi\right| \leq \tau(\varepsilon),
$$

pour tout $i, j$ distincts dans $\{1, \ldots, k\}$

$$
\begin{aligned}
& \left|d\left(x_{i}, x_{j}\right)-\frac{\pi}{2}\right| \leq \tau(\varepsilon), \\
& \left|d\left(x_{i}, y_{j}\right)-\frac{\pi}{2}\right| \leq \tau(\varepsilon), \\
& \left|d\left(y_{i}, y_{j}\right)-\frac{\pi}{2}\right| \leq \tau(\varepsilon) .
\end{aligned}
$$

De plus, ces points vérifient $f_{i}\left(x_{i}\right)=\sup _{M} f_{i}$ et $f_{i}\left(y_{i}\right)=\inf _{M} f_{i}$ avec $\left(f_{i}\right)_{1 \leq i \leq k}$ une famille orthogonale de fonctions propres associées à $\left(\lambda_{i}(M)\right)_{1 \leq i \leq k}$ et normalisées par (2).

Ces couples de points correspondent dans le cas modèle, aux couples formés de $k$ vecteurs $\left(e_{i}\right)_{1 \leq i \leq k}$ de la base canonique de $\mathbb{R}^{n+1}$ et des $k$ vecteurs opposés $\left(-e_{i}\right)_{1 \leq i \leq k}$.

Preuve. Soit $\left(x_{i}\right)_{1 \leq i \leq k}$ et $\left(y_{i}\right)_{1 \leq i \leq k}$ définis par

$$
f_{i}\left(x_{i}\right)=\sup _{M} f_{i} \text { et } f_{i}\left(y_{i}\right)=\inf _{M} f_{i}
$$

avec les fonctions $\left(f_{i}\right)_{1 \leq i \leq k}$ définies comme ci-dessus. D'après la proposition 3.2, pour tout $i$ dans $\{1, \ldots, k\}$, on a

$$
d\left(x_{i}, y_{i}\right)>\pi-\psi(\varepsilon) .
$$

Nous allons montrer l'existence d'une fonction $\tau(\varepsilon)$, telle que pour tout $i, j$ comme dans l'énoncé,

$$
\left|d\left(x_{i}, x_{j}\right)-\frac{\pi}{2}\right| \leq \tau(\varepsilon) .
$$

Admettons provisoirement ce résultat, on en déduit les autres estimations à l'aide d'un lemme sur la fonction «excess », dû à K. Grove et P. Petersen. 
Lemme 3.5 ([12]). Il existe une fonction $\tau(\varepsilon)$ telle que pour tout élément $(M, g)$ de $\mathcal{M}_{\mathrm{n}}$ et pour tout $p, q$ dans $M$ vérifiant

$$
d(p, q) \geq \pi-\varepsilon
$$

on $a$

$$
e_{p, q}(M)=\sup _{x \in M}(d(p, x)+d(q, x)-d(p, q)) \leq \tau(\varepsilon) .
$$

D'après ce lemme et $(21)$, il existe une fonction $\tau_{2}(\varepsilon)$ telle que pour tout $i, j$ distincts dans $\{1, \ldots, k\}$,

$$
\left|d\left(x_{j}, y_{j}\right)-d\left(x_{i}, x_{j}\right)-d\left(x_{i}, y_{j}\right)\right| \leq \tau_{2}(\varepsilon),
$$

donc par (21) et (22), il existe une fonction $\tau_{3}(\varepsilon)$ telle que

$$
\left|d\left(x_{i}, y_{j}\right)-\frac{\pi}{2}\right| \leq \tau_{3}(\varepsilon) .
$$

On déduit de manière similaire l'estimation sur $d\left(y_{i}, y_{j}\right)$.

Démontrons maintenant l'estimation (22). Fixons $i, j$ distincts dans $\{1, \ldots, k\}$. Notons

$$
h=f_{i} f_{j}+\left\langle d f_{i}, d f_{j}\right\rangle .
$$

Par hypothèse sur les fonctions $\left(f_{i}\right)_{1 \leq i \leq k}$,

$$
\frac{1}{\operatorname{vol~M}} \int_{\mathrm{M}} h=0
$$

Calculons la différentielle de $h$.

$$
\begin{aligned}
d h & =f_{j} d f_{i}+f_{i} d f_{j}+\operatorname{Hess} f_{i}\left(\nabla f_{j}, \cdot\right)+\operatorname{Hess} f_{j}\left(\nabla f_{i}, \cdot\right), \\
d h & =\left(\operatorname{Hess} f_{i}+f_{i} g\right)\left(\nabla f_{j}, \cdot\right)+\left(\operatorname{Hess} f_{j}+f_{j} g\right)\left(\nabla f_{i}, \cdot\right) .
\end{aligned}
$$

Donc

$$
|d h|^{2} \leq 2\left(\left\|\operatorname{Hess} f_{i}+f_{i} g\right\|^{2} \times\left\|\nabla f_{j}\right\|^{2}+\left\|\operatorname{Hess} f_{j}+f_{j} g\right\|^{2} \times\left\|\nabla f_{i}\right\|^{2}\right) .
$$

Or par la proposition 1.4, il existe une fonction $\tau_{4}(\varepsilon)$ telle que pour tout $s$ dans $\{1, \ldots, k\}$,

$$
\| \text { Hess } f_{s}+f_{s} g \|_{L^{2}} \leq \tau_{4}(\varepsilon) .
$$

Par (4), on en déduit l'existence d'une fonction $\tau_{5}(\varepsilon)$ telle que

$$
\frac{1}{\operatorname{vol~M}} \int_{\mathrm{M}}|d h|^{2} \leq \tau_{5}(\varepsilon)
$$


En appliquant l'inégalité de Poincaré à la fonction $h$ de moyenne nulle, on obtient, puisque $\lambda_{1}(M) \geq n$, l'existence d'une fonction $\tau_{6}(\varepsilon)$ telle que

$$
\frac{1}{\operatorname{vol~M}} \int_{\mathrm{M}} h^{2} \leq \tau_{6}(\varepsilon) \text {. }
$$

On déduit alors d'un corollaire du théorème de Bishop-Gromov [11, remarque 2.8], l'existence de fonctions $\tau_{7}(\varepsilon)$ et $R(\varepsilon)$ telles que pour tout $x$ dans $M$, il existe $\tilde{x}$ tel que $d(x, \tilde{x})<R(\varepsilon)$ et

$$
|h(\tilde{x})| \leq \tau_{7}(\varepsilon) .
$$

Pour $x=x_{j}$, la proposition 1.10 implique l'existence d'une constante $C^{\prime}(n)$ telle que

$$
\left|\nabla f_{j}\right|\left(\tilde{x}_{j}\right) \leq C^{\prime}(n) R(\varepsilon)
$$

puisque $f_{j}\left(x_{j}\right)=\sup _{M} f_{j}$. On en déduit qu'il existe une fonction $\tau_{8}(\varepsilon)$ telle que

$$
\left|h\left(\tilde{x}_{j}\right)-f_{i}\left(\tilde{x}_{j}\right) f_{j}\left(\tilde{x}_{j}\right)\right| \leq \tau_{8}(\varepsilon) .
$$

Mais, par la proposition 3.2

$$
\left\|\cos d_{x_{j}}-f_{j}\right\|_{L^{\infty}} \leq \tau_{9}(\varepsilon)
$$

d'où

$$
\left|f_{j}\left(\tilde{x}_{j}\right)-1\right| \leq C(n) R(\varepsilon)+\tau_{9}(\varepsilon) .
$$

Par conséquent, il existe une fonction $\tau_{10}(\varepsilon)$ telle que

$$
\left|f_{i}\left(\tilde{x}_{j}\right)\right| \leq \tau_{10}(\varepsilon) .
$$

On termine la preuve en appliquant de nouveau la proposition 3.2.

\section{Proximité de Gromov-Hausdorff}

Dans cette partie, nous montrons le

Théorème 4.1. Soit $k$ dans $\{2, \ldots, n+1\}$. Il existe une fonction $\tau(\varepsilon)$ telle que tout élément $(M, g)$ de $\mathcal{M}_{\mathrm{n}}$ vérifiant $\lambda_{k}(M) \leq n+\varepsilon$, possède un sous-ensemble $A \subset M$ presque convexe tel que $d_{G H}\left(A, S^{k-1}\right) \leq \tau(\varepsilon)$.

Nous renvoyons à la proposition 4.5 pour la définition de la presque convexité. Fixons $k$ dans $\{2, \ldots, n+1\}$. Notons $F=\left(f_{1}, \ldots, f_{k}\right)$ et $\Phi=\frac{F}{\sqrt{f_{1}^{2}+\cdots+f_{k}^{2}}}$ où les fonctions $\left(f_{i}\right)_{1 \leq i \leq k}$ sont les fonctions propres associées aux valeurs propres 
$\left(\lambda_{i}(M)\right)_{1 \leq i \leq k}$ et normalisées par (2). Sur la sphère canonique, les fonctions coordonnées (qui forment une base de fonctions propres de valeur propre $n$ ) fournissent un plongement isométrique d'une partie de $\left(\mathbb{S}^{n}, g_{\text {can }}\right)$ sur $\mathbb{S}^{k-1} \subset \mathbb{R}^{k}$ :

$$
\begin{aligned}
\left\{x \in \mathbb{S}^{n} ; X_{1}^{2}+\cdots+X_{k}^{2}=1\right\} & \longrightarrow \mathbb{S}^{k-1} \\
x & \longmapsto\left(X_{1}, \ldots, X_{k}\right)(x) .
\end{aligned}
$$

Nous allons montrer que l'application $\Phi$ restreinte à une partie $A_{k}$ convenable est une $\tau(\varepsilon)$ approximation de Gromov-Hausdorff, c'est-à-dire que pour tout $X$ dans $\mathbb{S}^{k-1}$, il existe $y$ dans $A_{k}$ tel que $d_{\mathbb{S}^{k-1}}(X, \Phi(y))<\tau(\varepsilon)$ et pour tout $x, y$ dans $A_{k},\left|d(x, y)-d_{\mathbb{S}^{k-1}}(\Phi(x), \Phi(y))\right|<\tau(\varepsilon)\left(d_{\mathbb{S}^{k-1}}\right.$ désigne la distance induite par la métrique canonique). Nous montrerons que pour une fonction $\eta(\varepsilon)$ bien choisie, $A_{k}=\left\{x \in M ;\left|\left(f_{1}^{2}+\cdots+f_{k}^{2}\right)(x)-1\right|<\eta(\varepsilon)\right\}$ convient.

Par choix de la partie $A_{k}$ et par uniforme continuité de la fonction arccos, il suffit pour démontrer le théorème 4.1 , de prouver l'existence d'une fonction $\tau(\varepsilon)$ pour laquelle la fonction $F$ vérifie les propriétés suivantes :

- une propriété de « $\tau(\varepsilon)$-presque surjectivité » : pour tout $X$ dans $\mathbb{S}^{k-1}$, il existe $x$ dans $A_{k}$ tel que

$$
\|F(x)-X\|_{\mathbb{R}^{k}} \leq \tau(\varepsilon),
$$

- une propriété de « $\tau(\varepsilon)$-proximité métrique » : pour tout $x, y$ dans $A_{k}$

$$
\left|\cos d(x, y)-\langle F(x), F(y)\rangle_{\mathbb{R}^{k}}\right| \leq \tau(\varepsilon),
$$

où $\langle., .\rangle_{\mathbb{R}^{k}}$ désigne le produit scalaire euclidien dans $\mathbb{R}^{k}$ et $\|\cdot\|_{\mathbb{R}^{k}}$ la norme associée.

La démonstration de (23) fait l'objet du prochain paragraphe, nous en déduirons (24) dans le paragraphe suivant.

4.1. Démonstration de la «presque surjectivité». Soit $(M, g)$ un élément de $\mathcal{M}_{\mathrm{n}}$, $s$ un entier non nul et $\eta$ un nombre réel positif. Soit $f_{1}, \ldots, f_{s}$ une famille orthogonale de fonctions propres sur $M$ associées à $\left(\lambda_{i}(M)\right)_{1 \leq i \leq s}$ et normalisées par (2). On note

$$
A_{s}^{\eta}=\left\{x \in M ;\left|f_{1}^{2}(x)+\cdots+f_{s}^{2}(x)-1\right|<\eta\right\} .
$$

Proposition 4.2. Soit $k$ dans $\{1, \ldots, n+1\}$. Il existe des fonctions $\eta(\varepsilon)$ et $\tau(\varepsilon)$ telles que pour tout élément $(M, g)$ de $\mathcal{M}_{\mathrm{n}}$ pour lequel $\lambda_{k}(M) \leq n+\varepsilon$, l'ensemble $A_{k}^{\eta(\varepsilon)}$ vérifie une propriété de $\tau(\varepsilon)$-presque surjectivité.

Preuve. La preuve repose sur une récurrence finie. Lorsque $k=1$, la proposition 4.2 est une conséquence directe de la proposition 3.2. Fixons $k$ dans $\{2, \ldots, n+1\}$ et $m$ dans $\{1, \ldots, k-1\}$. Dans la suite de la démonstration, on identifie $\left\{x \in \mathbb{S}^{k-1} ; x=\right.$ $\left.\left(a_{1}, \ldots, a_{m}, 0, \ldots, 0\right)\right\}$ avec $\mathbb{S}^{m-1}$. La preuve de la proposition est une conséquence du lemme suivant. 
Lemme 4.3. Supposons qu'il existe des fonctions $\eta_{m}(\varepsilon)$ et $\psi_{m}(\varepsilon)$ telles que pour tout $X$ dans $\mathbb{S}^{m-1}$, il existe x dans $A_{m}^{\eta_{m}(\varepsilon)}$ tel que

$$
\left\|\left(f_{1}, \ldots, f_{m}\right)(x)-X\right\|_{\mathbb{R}^{m}} \leq \psi_{m}(\varepsilon)
$$

alors il existe des fonctions $\eta_{m+1}(\varepsilon)$ et $\psi_{m+1}(\varepsilon)$ telles que pour tout $X$ dans $\mathbb{S}^{m}$, il existe x dans $A_{m+1}^{\eta_{m+1}(\varepsilon)}$ tel que

$$
\left\|\left(f_{1}, \ldots, f_{m+1}\right)(x)-X\right\|_{\mathbb{R}^{m+1}} \leq \psi_{m+1}(\varepsilon) .
$$

Démontrons le lemme 4.3. Commençons par remarquer que pour toute fonction $\eta(\varepsilon)$, il existe une fonction $\theta(\varepsilon)$ telle que pour tout $s$ dans $\{1, \ldots, k-1\}$,

$$
A_{s}^{\eta(\varepsilon)} \subset A_{s+1}^{\theta(\varepsilon)},
$$

c'est-à-dire que $f_{s+1}\left(A_{s}^{\eta(\varepsilon)}\right)$ est presque réduit à $\{0\}$. Ce résultat est une conséquence directe d'un lemme démontré par P. Petersen [17, lemme 3.3].

Lemme 4.4 ([17]). Il existe une fonction $\tau(\varepsilon)$ telle que pour tout élément $(M, g)$ dans $\mathcal{M}_{\mathrm{n}}$ vérifiant $\lambda_{k}(M) \leq n+\varepsilon$, on a pour tout $x$ dans $M$

$$
f_{1}^{2}(x)+\cdots+f_{k}^{2}(x) \leq 1+\tau(\varepsilon),
$$

où $\left(f_{i}\right)_{1 \leq i \leq k}$ est une famille orthogonale de fonctions propres de $M$, de valeurs propres $\left(\lambda_{i}(M)\right)_{1 \leq i \leq k}$ et normalisées par $\frac{1}{\mathrm{vol} M} \int_{\mathrm{M}} f_{i}^{2}=\frac{1}{n+1}$.

Soit $Y=\left(\cos s_{1}, \ldots, \cos s_{m+1}\right)$ dans $\mathbb{S}^{m}$. Il faut distinguer les cas $\left|\sin s_{m+1}\right|<$ $\mu(\varepsilon)$ et $\left|\sin s_{m+1}\right| \geq \mu(\varepsilon)$, où $\mu(\varepsilon)$ vérifie $\lim _{\varepsilon \rightarrow 0} \mu(\varepsilon)=0$ et sera défini plus loin.

Supposons $\left|\sin s_{m+1}\right|<\mu(\varepsilon)$. Dans ce cas, comme $d\left(x_{i}, x_{m+1}\right)$ est proche de $\frac{\pi}{2}$ et $d\left(x_{i}, y_{m+1}\right)$ est proche de $\frac{\pi}{2}$ pour tout $i$ dans $\{1, \ldots, m\}$, la proposition 3.2 implique l'existence d'une fonction $\tau_{2}(\varepsilon)$ telle que

$$
\left\|\left(f_{1}, \ldots, f_{m+1}\right)(\alpha)-Y\right\|_{\mathbb{R}^{m+1}} \leq \tau_{2}(\varepsilon),
$$

avec $\alpha=x_{m+1}$ si $s_{m+1}$ est proche de 0 et $\alpha=y_{m+1}$ sinon.

On suppose maintenant que $\left|\sin s_{m+1}\right| \geq \mu(\varepsilon)$ et que $s_{m+1} \leq \frac{\pi}{2}$. Le cas $s_{m+1}>\frac{\pi}{2}$ sera traité plus loin. On définit

$$
X=\left(\frac{\cos s_{1}}{\sin s_{m+1}}, \ldots, \frac{\cos s_{m}}{\sin s_{m+1}}\right) \in \mathbb{S}^{m-1} .
$$

Par hypothèse de récurrence, il existe $x_{0} \in A_{m}^{\eta_{m}(\varepsilon)}$ telle que

$$
\left\|\left(f_{1}, \ldots, f_{m}\right)\left(x_{0}\right)-X\right\|_{\mathbb{R}^{m}} \leq \psi_{m}(\varepsilon) .
$$


D'après (25), il existe une fonction $\theta(\varepsilon)$ telle que $A_{m}^{\eta_{m}(\varepsilon)} \subset A_{m+1}^{\theta(\varepsilon)}$. D'après la proposition 3.2, $f_{m+1}$ est proche en norme $L^{\infty}$ de $\cos d_{x_{m+1}}$ donc comme $x_{0}$ appartient à $A_{m}^{\eta_{m}(\varepsilon)}$, l'équation (25) implique l'existence d'une fonction $\tau_{3}(\varepsilon)$ telle que

$$
\left|\cos d_{x_{m+1}}\left(x_{0}\right)\right| \leq \tau_{3}(\varepsilon) .
$$

Par conséquent, par l'inégalité des accroissements finis, on a

$$
\left|\frac{\pi}{2}-d_{x_{m+1}}\left(x_{0}\right)\right| \leq \tau_{3}(\varepsilon) .
$$

En appliquant le lemme 1.6 aux fonctions $f_{i}$ (pour $i$ dans $\{1, \ldots, m+1\}$ ) au voisinage des points $x_{0}$ et $x_{m+1}$, on en déduit qu'il existe $\tilde{x}_{0}$ avec $d\left(\tilde{x}_{0}, x_{0}\right)<r(\varepsilon)$ et $\tilde{x}_{m+1}$ avec $d\left(x_{m+1}, \tilde{x}_{m+1}\right)<r(\varepsilon)$, tels que si on note $\gamma$ l'unique géodésique minimisante reliant $\tilde{x}_{m+1}$ à $\tilde{x}_{0}$ et $u_{i}=f_{i} \circ \gamma$ (pour $i \in\{1, \ldots, m+1\}$ ) alors

$$
\int_{0}^{d\left(\tilde{x}_{0}, \tilde{x}_{m+1}\right)}\left|u_{i}^{\prime \prime}(t)+u_{i}(t)\right|^{2} d t \leq \tau_{4}(\varepsilon) .
$$

Pour $i$ dans $\{1, \ldots, m\}$, les conditions aux bords sont

$$
u_{i}(0)=f_{i}\left(\tilde{x}_{m+1}\right)
$$

et

$$
u_{i}\left(d\left(\tilde{x}_{m+1}, \tilde{x}_{0}\right)\right)=f_{i}\left(\tilde{x}_{0}\right)
$$

Or, d'une part

$$
\left|f_{i}\left(\tilde{x}_{m+1}\right)\right| \leq\left|f_{i}\left(\tilde{x}_{m+1}\right)-f_{i}\left(x_{m+1}\right)\right|+\left\|f_{i}-\cos d_{x_{i}}\right\|_{L^{\infty}}+\left|\cos d_{x_{i}}\left(x_{m+1}\right)\right|,
$$

donc

$$
\left|f_{i}\left(\tilde{x}_{m+1}\right)\right| \leq C(n) r(\varepsilon)+\tau_{5}(\varepsilon)+\tau_{6}(\varepsilon)
$$

et d'autre part

$$
\left|f_{i}\left(\tilde{x}_{0}\right)-\frac{\cos s_{i}}{\sin s_{m+1}}\right| \leq\left|f_{i}\left(\tilde{x}_{0}\right)-f_{i}\left(x_{0}\right)\right|+\left|f_{i}\left(x_{0}\right)-\frac{\cos s_{i}}{\sin s_{m+1}}\right|
$$

donc

$$
\left|f_{i}\left(\tilde{x}_{0}\right)-\frac{\cos s_{i}}{\sin s_{m+1}}\right| \leq C(n) r(\varepsilon)+\psi_{m}(\varepsilon) .
$$

Pour $i$ dans $\{1, \ldots, m\}$, notons $\bar{u}_{i}(t)=\frac{\cos s_{i}}{\sin s_{m+1}} \sin (t)$. En utilisant (27), on en déduit

$$
\left|\bar{u}_{i}\left(\frac{\pi}{2}\right)-\bar{u}_{i}\left(d\left(\tilde{x}_{m+1}, \tilde{x}_{0}\right)\right)\right| \leq \frac{\tau_{3}(\varepsilon)+2 r(\varepsilon)}{\mu(\varepsilon)} .
$$


On fixe $\mu(\varepsilon)=\sqrt{\tau_{3}(\varepsilon)+2 r(\varepsilon)}$. D'après (28) et (29), il existe une fonction $\tau_{7}(\varepsilon)$ telle que

$$
\left|u_{i}(0)-\bar{u}_{i}(0)\right| \leq \tau_{7}(\varepsilon)
$$

et

$$
\left|u_{i}\left(d\left(\tilde{x}_{m+1}, \tilde{x}_{0}\right)\right)-\bar{u}_{i}\left(d\left(\tilde{x}_{m+1}, \tilde{x}_{0}\right)\right)\right| \leq \tau_{7}(\varepsilon) .
$$

D'après (27), on peut supposer $\varepsilon$ assez petit pour que $l=d\left(\tilde{x}_{m+1}, \tilde{x}_{0}\right)$ vérifie l'hypothèse du lemme 1.8, par conséquent en appliquant ce lemme aux fonctions $u_{i}$ et $\bar{u}_{i}$, on obtient l'existence d'une fonction $\tau_{8}(\varepsilon)$ telle que pour tout $i$ dans $\{1, \ldots, m\}$ et pour tout $t$ dans $\left[0, d\left(\tilde{x}_{m+1}, \tilde{x}_{0}\right)\right]$

$$
\left|\left(f_{i} \circ \gamma\right)(t)-\frac{\cos s_{i}}{\sin s_{m+1}} \sin (t)\right| \leq \tau_{8}(\varepsilon) .
$$

La proposition 3.2 permet d'estimer $f_{m+1}$, on obtient

$$
\left|f_{m+1}(\gamma(t))-\cos t\right| \leq \tau_{5}(\varepsilon)+r(\varepsilon) .
$$

En combinant ces résultats, on en déduit l'existence d'une fonction $\tau_{9}(\varepsilon)$ telle que pour tout $t$ dans $\left[0, d\left(\tilde{x}_{m+1}, \tilde{x}_{0}\right)\right]$,

$$
\begin{aligned}
& \|\left(f_{1}, \ldots, f_{m+1}\right)(\gamma(t)) \\
& \quad-\left(\cos s_{1}, \ldots, \cos s_{m}, 0\right) \frac{\sin t}{\sin s_{m+1}}+(0, \ldots, 0, \cos t) \|_{\mathbb{R}^{m+1}} \leq \tau_{9}(\varepsilon) .
\end{aligned}
$$

D'où, comme on suppose $s_{m+1} \leq \frac{\pi}{2}$, on obtient pour

$$
\begin{gathered}
T=\min \left\{d\left(\tilde{x}_{m+1}, \tilde{x}_{0}\right), s_{m+1}\right\} \\
\left\|\left(f_{1}, \ldots, f_{m+1}\right)(\gamma(T))-\left(\cos s_{1}, \ldots, \cos s_{m}, \cos s_{m+1}\right)\right\|_{\mathbb{R}^{m+1}} \\
\leq \tau_{9}(\varepsilon)+\|\left(\left(\cos s_{1}, \ldots, \cos s_{m}, 0\right)\left(\frac{\sin T}{\sin s_{m+1}}-1\right)\right. \\
+(0, \ldots, 0,1)\left(\cos T-\cos s_{m+1}\right) \|_{\mathbb{R}^{m+1}} .
\end{gathered}
$$

Or comme par (27)

$$
d\left(\tilde{x}_{m+1}, \tilde{x}_{0}\right) \geq \frac{\pi}{2}-\tau_{3}(\varepsilon)-2 r(\varepsilon),
$$

la proposition est démontrée dans ce cas. Si $s_{m+1} \geq \frac{\pi}{2}$, il suffit de remplacer $x_{m+1}$ par $y_{m+1}$ dans tout ce qui précède. 
4.2. Propriété de «proximité métrique». Soit $k$ dans $\{2, \ldots, n+1\}$. On conserve la notation $A_{k}^{\eta(\varepsilon)}$ pour la partie introduite dans la proposition 4.2. Dans cette partie, nous montrons que $F=\left(f_{1}, \ldots, f_{k}\right)$ vérifie la propriété de $\tau(\varepsilon)$-proximité métrique, dont nous rappelons la définition :

Il existe une fonction $\tau(\varepsilon)$ telle que tout élément $(M, g)$ de $\mathcal{M}_{\mathrm{n}}$ tel que $\lambda_{k}(M) \leq$ $n+\varepsilon$, vérifie pour tout $x, y$ dans $A_{k}^{\eta(\varepsilon)}$

$$
\left|\cos d(x, y)-\langle F(x), F(y)\rangle_{\mathbb{R}^{k}}\right| \leq \tau(\varepsilon),
$$

ce qui termine la preuve du théorème 4.1. Nous démontrons également que tout élément $(M, g)$ de $\mathcal{M}_{\mathrm{n}}$ tel que $\lambda_{n+1}(M) \leq n+\varepsilon$, vérifie

$$
d_{G H}\left(M, \mathbb{S}^{n}\right) \leq \tau(\varepsilon) .
$$

Enfin nous démontrons que la partie $A_{k}^{\eta(\varepsilon)}$ est «presque convexe» au sens de la proposition ci-dessous.

Proposition 4.5. Il existe des fonctions $\eta^{\prime}(\varepsilon)$ et $\tau(\varepsilon)$ telles que tout élément $(M, g)$ de $\mathcal{M}_{\mathrm{n}}$ pour lequel $\lambda_{k}(M) \leq n+\varepsilon$, vérifie la propriété suivante. Pour tout $x, y$ dans $A_{k}^{\eta(\varepsilon)}$,

$$
d_{A_{k}^{\eta^{\prime}(\varepsilon)}}(x, y) \leq d(x, y)+\tau(\varepsilon)
$$

où $d_{A_{k}^{\eta^{\prime}(\varepsilon)}}$ désigne la distance intrinsèque de l'ouvert $A_{k}^{\eta^{\prime}(\varepsilon)}$ et $\eta^{\prime}(\varepsilon)$ vérifie l'inégalité $\eta^{\prime}(\varepsilon) \geq \eta(\varepsilon)$.

Commençons par montrer comment la démonstration de la propriété (32) se ramène à la démonstration que nous allons donner de la propriété (31). Sous l'hypothèse $\lambda_{n+1}(M) \leq n+\varepsilon$, la proposition 4.2 montre que la variété $M$ contient une partie qui est $\tau(\varepsilon)$-presque surjective sur $\mathbb{S}^{n}$. Il suffit donc de prouver que cette application vérifie la propriété de $\tau(\varepsilon)$-proximité métrique pour une fonction $\tau(\varepsilon)$ convenable. Or, d'après un résultat de P. Petersen [17, lemme 5.2], il existe une fonction $R(\varepsilon)$ telle que

$$
M=A_{n+1}^{R(\varepsilon)},
$$

avec $R(\varepsilon) \geq \eta(\varepsilon)$ (où $\eta(\varepsilon)$ a été introduit dans la proposition 4.2). Pour démontrer la propriété (32), il suffit donc de démontrer la propriété de proximité métrique (31) pour toute partie $A_{k}^{R(\varepsilon)}$ avec $R(\varepsilon) \geq \eta(\varepsilon)$.

Plan de la preuve de l'estimation (31). Dans une première partie, nous démontrons que tout point de $A_{k}^{R(\varepsilon)}$ (avec $R(\varepsilon) \geq \eta(\varepsilon)$ ) admet un presque antipode dans $A_{k}^{R(\varepsilon)}$. Nous démontrons également la proposition 4.5. Ensuite grâce au lemme 2.2, nous montrons que pour tout $x, y$ dans $A_{k}^{R(\varepsilon)}, \cos d(x, y)$ est proche de $\sum_{i=1}^{k} a_{i}(x) f_{i}(y)$, 
avec $\left(a_{i}(x)\right)_{i \geq 0}$, les coefficients de Fourier de $\cos d_{x}$. Nous montrons ensuite que $a_{i}(x)$ est proche de $f_{i}(x)$ (il y a égalité dans le cas de la sphère), ce qui permet de conclure.

4.2.1. Propriétés des ensembles $\boldsymbol{A}_{\boldsymbol{k}}^{\boldsymbol{R}(\boldsymbol{\varepsilon})}$. Soit $k$ dans $\{2, \ldots, n+1\}$ et $A_{k}^{R(\varepsilon)}$ avec

$$
R(\varepsilon) \geq \eta(\varepsilon)
$$

fixé.

Sur la sphère canonique, l'ensemble $\left\{x \in \mathbb{S}^{n} ; X_{1}^{2}(x)+\cdots+X_{k}^{2}(x)=1\right\}$ où les fonctions $\left(X_{i}\right)_{1 \leq i \leq k}$ sont les $k$ premières fonctions coordonnées, est un équateur de dimension $k-1$. La fonction $X_{1}^{2}+\cdots+X_{k}^{2}$ définie sur $\mathbb{S}^{n}$ atteint son maximum sur cet équateur, son gradient est donc nul sur cet ensemble.

Le lemme suivant est une généralisation de ce fait au «presque équateur» $A_{k}^{R(\varepsilon)}$, dans le cas où la variété $(M, g)$ admet $k$ valeurs propres de $n$.

Lemme 4.6. Il existe une fonction $\tau(\varepsilon)$, telle que pour toute fonction $\theta(\varepsilon)$ et pour tout élément $(M, g)$ dans $\mathcal{M}_{\mathrm{n}}$, vérifiant $\lambda_{k}(M) \leq n+\varepsilon$, on a pour tout $x$ dans $A_{k}^{\theta(\varepsilon)}$ l'estimation

$$
\left|\nabla\left(\sum_{i=1}^{k} f_{i}^{2}\right)\right|(x) \leq 4(1+\theta(\varepsilon))(\tau(\varepsilon)+\theta(\varepsilon)),
$$

avec $\left(f_{i}\right)_{1 \leq i \leq k}$ une famille orthogonale de fonctions propres associées à $\left(\lambda_{i}(M)\right)_{1 \leq i \leq k}$ et normalisées par (2).

Preuve. Fixons un point $x_{0}$ de $A_{k}^{\theta(\varepsilon)}$ et considérons les coefficients $a_{i}=\frac{f_{i}\left(x_{0}\right)}{\sqrt{\sum_{i=1}^{k} f_{i}^{2}\left(x_{0}\right)}}$ pour $i$ dans $\{1, \ldots, k\}$. On note $f=\sum_{i=1}^{k} a_{i} f_{i}$. En développant le terme $|\nabla f|^{2}$, on obtient

$$
|\nabla f|^{2}(x)=\sum_{1 \leq i, j \leq k} a_{i} a_{j}\left\langle\nabla f_{i}(x), \nabla f_{j}(x)\right\rangle
$$

D'où, en $x_{0}$

$$
|\nabla f|^{2}\left(x_{0}\right)=\frac{1}{4 \sum_{i=1}^{k} f_{i}^{2}\left(x_{0}\right)}\left|\nabla\left(\sum_{i=1}^{k} f_{i}^{2}\right)\left(x_{0}\right)\right|^{2} .
$$

Par conséquent en appliquant la proposition 1.1 à $f$ au point $x_{0}$, on obtient

$$
\sum_{i=1}^{k} f_{i}^{2}\left(x_{0}\right)+\frac{1}{4 \sum_{i=1}^{k} f_{i}^{2}\left(x_{0}\right)}\left|\nabla\left(\sum_{i=1}^{k} f_{i}^{2}\right)\left(x_{0}\right)\right|^{2} \leq 1+\tau(\varepsilon) .
$$

Or $x_{0}$ appartient à $A_{k}^{\theta(\varepsilon)}$ entraine $1+\theta(\varepsilon) \geq \sum_{i=1}^{k} f_{i}^{2}\left(x_{0}\right) \geq 1-\theta(\varepsilon)$, d'où le résultat. 
Ce lemme permet de démontrer que tout point de $A_{k}^{R(\varepsilon)}$ admet un presque antipode.

Lemme 4.7. Il existe une fonction $\delta(\varepsilon)$ (vérifiant $\lim _{\varepsilon \rightarrow 0} \frac{\delta(\varepsilon)}{\varepsilon}=+\infty$ ) telle que pour tout élément $(M, g)$ de $\mathcal{M}_{\mathrm{n}}$ vérifiant $\lambda_{k}(M) \leq n+\varepsilon$ et pour tout $x$ dans $A_{k}^{R(\varepsilon)}$, il existe y dans $A_{k}^{R(\varepsilon)}$ avec

$$
d(x, y)>\pi-\delta(\varepsilon) .
$$

Preuve. Dans le cas de la sphère, le point antipodal d'un point $X$ de $\mathbb{S}^{n}$ est $-X$, ce qui suggère le «candidat» à être un presque antipode de $x$ appartenant à $A_{k}^{R(\varepsilon)}$. Soit $x$ dans $A_{k}^{R(\varepsilon)}$, notons $\alpha=\left\|\left(f_{1}, \ldots, f_{k}\right)(x)\right\|_{\mathbb{R}^{k}}$, l'hypothèse $x$ appartient à $A_{k}^{R(\varepsilon)}$ implique

$$
(1-R(\varepsilon))^{\frac{1}{2}} \leq \alpha \leq(1+R(\varepsilon))^{\frac{1}{2}} .
$$

Par la proposition 4.2 de presque surjectivité, il existe $y$ appartenant à $A_{k}^{\eta(\varepsilon)}$ tel que

$$
\left\|\left(f_{1}, \ldots, f_{k}\right)(y)+\frac{1}{\alpha}\left(f_{1}, \ldots, f_{k}\right)(x)\right\| \leq \tau(\varepsilon) .
$$

En appliquant le lemme 1.6 aux fonctions $\left(f_{i}\right)_{1 \leq i \leq k}$, on déduit l'existence de $\tilde{x}$ et $\tilde{y}$ vérifiant

$$
d(x, \tilde{x}) \leq r(\varepsilon), d(y, \tilde{y}) \leq r(\varepsilon)
$$

et en notant $\gamma_{\tilde{x} \tilde{y}}$ l'unique géodésique minimisante reliant $\tilde{x}$ à $\tilde{y}$, on a pour tout $i$ dans $\{1, \ldots, k\}$,

$$
\int_{0}^{d(\tilde{x}, \tilde{y})}\left|\left(f_{i} \circ \gamma\right)^{\prime \prime}(t)+\left(f_{i} \circ \gamma\right)(t)\right|^{2} d t \leq \tau^{\prime}(\varepsilon) .
$$

D'autre part, par le lemme 4.6

$$
\left|\nabla\left(f_{1}^{2}+\cdots+f_{k}^{2}\right)\right|(\tilde{x}) \leq \tau^{\prime \prime}(\varepsilon) .
$$

Notons $a_{i}=f_{i}(\tilde{x}), b_{i}=\left(f_{i} \circ \gamma_{\tilde{x} \tilde{y}}\right)^{\prime}(0)$ et $l=d(\tilde{x}, \tilde{y})$. On déduit de l'équation (35) et du lemme 1.9, l'existence d'une fonction $\tau_{2}(\varepsilon)$ telle que pour tout $i$ dans $\{1, \ldots, k\}$ et pour tout $t$ dans $[0, l]$,

$$
\begin{gathered}
\left|\left(f_{i} \circ \gamma_{\tilde{x} \tilde{y}}\right)(t)-\left(a_{i} \cos t+b_{i} \sin t\right)\right| \leq \tau_{2}(\varepsilon), \\
\left|\left(f_{i} \circ \gamma_{\tilde{x} \tilde{y}}\right)^{\prime}(t)-\left(-a_{i} \sin t+b_{i} \cos t\right)\right| \leq \tau_{2}(\varepsilon) .
\end{gathered}
$$

En appliquant l'inégalité de Cauchy-Schwartz, on obtient à l'aide de (36)

$$
\left|\sum_{i=1}^{k} a_{i} b_{i}\right|=\left|\frac{1}{2}\left(\left(\sum_{i=1}^{k} f_{i}^{2}\right) \circ \gamma_{\tilde{x} \tilde{y}}\right)^{\prime}(0)\right| \leq \frac{\tau^{\prime \prime}(\varepsilon)}{2} .
$$


Estimons maintenant $f_{i}(\tilde{y})$ pour $i$ dans $\{1, \ldots, k\}$.

$f_{i}(\tilde{y})+a_{i}=\left(f_{i}(\tilde{y})-f_{i}(y)\right)+\left(f_{i}(y)+\frac{f_{i}(x)}{\alpha}\right)+\left(-\frac{f_{i}(x)}{\alpha}+f_{i}(x)\right)+\left(-f_{i}(x)+f_{i}(\tilde{x})\right)$.

D'où, par définition de $y$ et par (34)

$$
\begin{aligned}
\left|f_{i}(\tilde{y})+a_{i}\right| \leq & 2 C(n) r(\varepsilon)+\tau(\varepsilon) \\
& +C(n) \max \left\{-(1+R(\varepsilon))^{-\frac{1}{2}}+1 ;-1+(1-R(\varepsilon))^{-\frac{1}{2}}\right\} .
\end{aligned}
$$

Donc, il existe une fonction $\tau_{3}(\varepsilon)$ telle que pour tout $i$ dans $\{1, \ldots, k\}$,

$$
\left|f_{i}(\tilde{y})+a_{i}\right| \leq \tau_{3}(\varepsilon) .
$$

En appliquant (37) avec $t=l$, on obtient

$$
a_{i} \cos l+b_{i} \sin l=-a_{i}+\delta_{i},
$$

avec $\left|\delta_{i}\right| \leq \tau_{3}(\varepsilon)$.

En multipliant (40) par $b_{i}$ et en sommant par rapport à $i$, on obtient

$$
\left(\sum_{i=1}^{k} a_{i} b_{i}\right) \cos l+\left(\sum_{i=1}^{k} b_{i}^{2}\right) \sin l+\sum_{i=1}^{k} a_{i} b_{i} \leq\left(\sum_{i=1}^{k} b_{i}^{2}\right)^{\frac{1}{2}} \delta^{\prime} .
$$

$\operatorname{avec} \delta^{\prime}=\sqrt{\sum_{i=1}^{k} \delta_{i}^{2}}$. Or $\left|b_{i}\right| \leq\left|\nabla f_{i}\right|$, donc la proposition 1.1 implique que $\sum_{i=1}^{k} b_{i}^{2}$ est bornée par une constante $C(n)$. D'autre part $\left|\delta^{\prime}\right| \leq(n+1)^{\frac{1}{2}} \tau_{3}(\varepsilon)$, donc on déduit de (38) l'existence d'une fonction $\tau_{4}(\varepsilon)$ telle que

$$
\left|\left(\sum_{i=1}^{k} b_{i}^{2}\right) \sin l\right| \leq \tau_{4}(\varepsilon),
$$

par conséquent, soit $|\sin l| \leq\left(\tau_{4}(\varepsilon)\right)^{\frac{1}{2}}$, soit $\sum_{i=1}^{k} b_{i}^{2} \leq\left(\tau_{4}(\varepsilon)\right)^{\frac{1}{2}}$.

Premier cas $:|\sin l| \leq\left(\tau_{4}(\varepsilon)\right)^{\frac{1}{2}}$. Comme $\left\|\left(f_{1}, \ldots, f_{k}\right)(x)\right\|_{\mathbb{R}^{k}}$ est proche de 1 , on en déduit que $\left(f_{1}, \ldots, f_{k}\right)(x)$ est presque égal à $-\left(f_{1}, \ldots, f_{k}\right)(y)$. Le gradient des fonctions propres étant borné (4), cela implique l'existence d'une constante $C^{\prime}(n)>0$ telle que

$$
d(x, y)>C^{\prime}(n)
$$

et donc $|\sin l| \leq\left(\tau_{4}(\varepsilon)\right)^{\frac{1}{2}}$ implique que $l$ est presque égal à $\pi$.

Deuxième cas : $\sum_{i=1}^{k} b_{i}^{2} \leq\left(\tau_{4}(\varepsilon)\right)^{\frac{1}{2}}$. Dans ce cas par (37), il existe une fonction $\tau_{5}(\varepsilon)$ telle que pour tout $i$ dans $\{1, \ldots, k\}$ et pour tout $t$ dans $[0, l]$,

$$
\left.\mid\left(f_{i} \circ \gamma_{\tilde{x} \tilde{y}}\right)(t)\right)-a_{i} \cos t \mid \leq \tau_{5}(\varepsilon),
$$

en appliquant cette formule avec $t=l$, on obtient par (39) que $\cos l$ est presque égal à -1 , d'où le résultat. 
À l'aide d'une légère modification de la preuve ci-dessus, nous sommes en mesure de démontrer la propriété de «presque convexité » de l'ensemble $A_{k}^{\eta(\varepsilon)}$ (proposition 4.5).

Preuve. Le début de la preuve est identique à celle du lemme 4.7. Soit $x, y$ dans $A_{k}^{\eta(\varepsilon)}$. En appliquant le lemme 1.6 aux fonctions $\left(f_{i}\right)_{1 \leq i \leq k}$, on déduit l'existence de $\tilde{x}$ et $\tilde{y}$ vérifiant $d(x, \tilde{x}) \leq r(\varepsilon), d(y, \tilde{y}) \leq r(\varepsilon)$ tels que, si on note $\gamma_{\tilde{x} \tilde{y}}$, l'unique géodésique minimisante reliant $\tilde{x}$ à $\tilde{y}$, on a pour tout $i$ dans $\{1, \ldots, k\}$,

$$
\int_{0}^{d(\tilde{x}, \tilde{y})}\left|\left(f_{i} \circ \gamma\right)^{\prime \prime}(t)+\left(f_{i} \circ \gamma\right)(t)\right|^{2} d t \leq \tau^{\prime}(\varepsilon) .
$$

Notons $a_{i}=f_{i}(\tilde{x}), b_{i}=\left(f_{i} \circ \gamma_{\tilde{x} \tilde{y}}\right)^{\prime}(0)$ et $l=d(\tilde{x}, \tilde{y})$. On en déduit comme précédemment (38) que pour tout $t$ dans $[0, l]$,

$$
\left.\mid\left(f_{i} \circ \gamma_{\tilde{x} \tilde{y}}\right)(t)\right)-\left(a_{i} \cos t+b_{i} \sin t\right) \mid \leq \tau_{2}(\varepsilon)
$$

avec

$$
\left|\sum_{i=1}^{k} a_{i} b_{i}\right| \leq \frac{\tau(\varepsilon)}{2}
$$

Par (4), il existe une constante $C(n)$ telle que

$$
\left\|\nabla\left(\sum_{i=1}^{k} f_{i}^{2}\right)\right\|_{L^{\infty}} \leq C(n) .
$$

Par conséquent, les hypothèses sur $x$ et $\tilde{x}$ impliquent

$$
d(x, \tilde{x})=d_{A_{k}^{\eta(\varepsilon)+C(n) r(\varepsilon)}}(x, \tilde{x}),
$$

de même pour $y$ et $\tilde{y}$. Notons $\eta_{2}(\varepsilon)=\eta(\varepsilon)+C(n) r(\varepsilon)$. Pour démontrer la proposition, il suffit donc de prouver qu'il existe des fonctions $\eta^{\prime}(\varepsilon)$ et $\psi(\varepsilon)$ telles que

$$
d_{A_{k}^{\eta^{\prime}(\varepsilon)}}(\tilde{x}, \tilde{y}) \leq d(\tilde{x}, \tilde{y})+\psi(\varepsilon) .
$$

Par construction $\tilde{y}$ appartient à $A_{k}^{\eta_{2}(\varepsilon)}$, c'est-à-dire

$$
\left|\sum_{i=1}^{k}\left(f_{i}^{2} \circ \gamma_{\tilde{x} \tilde{y}}\right)(l)-1\right| \leq \eta_{2}(\varepsilon) .
$$

Par ailleurs, grâce à (42) et (43), il existe une fonction $\tau(\varepsilon)$ telle que

$$
\left|\sum_{i=1}^{k}\left(f_{i}^{2} \circ \gamma_{\tilde{x} \tilde{y}}\right)(l)-\left(\left(\sum_{i=1}^{k} a_{i}^{2}\right) \cos ^{2} l+\left(\sum_{i=1}^{k} b_{i}^{2}\right) \sin ^{2} l\right)\right| \leq \tau(\varepsilon) .
$$


Or, comme $a_{i}=f_{i}(\tilde{x})$ et $\tilde{x}$ appartient à $A_{k}^{\eta_{2}(\varepsilon)}$, (44) et (45) impliquent l'existence d'une fonction $\tau_{2}(\varepsilon)$ telle que

$$
\left|\left(1-\sum_{i=1}^{k} b_{i}^{2}\right) \sin ^{2} l\right| \leq \tau_{2}(\varepsilon) .
$$

Par conséquent, soit $\left|\sum_{i=1}^{k} b_{i}^{2}-1\right| \leq \sqrt{\tau_{2}(\varepsilon)}$, soit $\sin ^{2} l \leq \sqrt{\tau_{2}(\varepsilon)}$. Supposons tout d'abord que $\left|\sum_{i=1}^{k} b_{i}^{2}-1\right| \leq \sqrt{\tau_{2}(\varepsilon)}$. Dans ce cas, on obtient en utilisant (42) et (43), l'existence d'une fonction $\tau_{3}(\varepsilon)$ telle que pour tout $t$ dans $[0, l]$,

$$
\left|\sum_{i=1}^{k}\left(f_{i}^{2} \circ \gamma_{\tilde{x} \tilde{y}}\right)(t)-1\right| \leq \tau_{3}(\varepsilon) .
$$

La proposition est démontrée dans ce premier cas. Supposons maintenant que $\sin ^{2} l \leq$ $\sqrt{\tau_{2}(\varepsilon)}$. Ce qui signifie que $d(\tilde{x}, \tilde{y})$ est proche de 0 ou de $\pi$. Si $d(\tilde{x}, \tilde{y})$ est proche de 0 , c'est immédiat. Si $d(\tilde{x}, \tilde{y})$ est presque égal à $\pi$ alors nécessairement, avec les notations de la proposition 3.4, il existe $i_{0}$ dans $\{1, \ldots, k\}$ tel que $\sin ^{2} d\left(x_{i_{0}}, \tilde{x}\right) \geq \sqrt{\tau_{2}(\varepsilon)}$ et $\sin ^{2} d\left(x_{i_{0}}, \tilde{y}\right) \geq \sqrt{\tau_{2}(\varepsilon)}$. Par conséquent, d'après le premier cas, la courbe $c$ formée de l'union des deux géodésiques minimisantes reliant $\tilde{x}$ à un point voisin de $x_{i_{0}}$ et ce dernier point à $\tilde{y}$ est contenue dans $A_{k}^{\tau_{3}(\varepsilon)}$. Le lemme 3.5 sur la fonction «excess » permet alors de conclure.

4.2.2. Démonstration de la propriété de «proximité métrique». Nous venons de montrer que tout point de $A_{k}^{R(\varepsilon)}$ admet un presque antipode. L'idée de la preuve consiste à utiliser la propriété des fonctions $\cos d_{p}$ pour $p$ admettant un presque antipode, établie dans le lemme 2.2. Cependant, on ne peut pas appliquer directement le lemme 2.2 avec la fonction $\delta(\varepsilon)$ introduite dans le lemme 4.7, puisqu'on voit facilement (par exemple dans la proposition 1.4) que les fonctions $\tau(\varepsilon)$ utilisées dans la preuve du lemme 4.7 sont supérieures à $\varepsilon$ et donc

$$
\lim _{\varepsilon \rightarrow 0} \frac{\delta(\varepsilon)}{\varepsilon}=+\infty \text {. }
$$

Pour contourner ce problème, on pose

$$
\bar{k}=\max \left\{i ; \lambda_{i} \leq n+\sqrt{\delta(\varepsilon)}\right\} .
$$

$\operatorname{Par}$ (46) et pour $\varepsilon$ assez petit, on a $\bar{k} \geq k$. D'après le lemme 2.2 appliqué avec $\eta=\delta(\varepsilon)$ et $\sqrt{\delta(\varepsilon)}$ et le lemme 4.7, il existe une fonction $\tau(\varepsilon)$ telle que pour tout $x$ dans $A_{k}^{R(\varepsilon)}$, il existe des coefficients $\left(\alpha_{i}(x)\right)_{i=1}^{\bar{k}}$ pour lesquels pour tout $z$ dans $M$,

$$
\left|\cos d_{x}(z)-\sum_{i=1}^{\bar{k}} \alpha_{i}(x) f_{i}(z)\right| \leq \tau(\varepsilon),
$$


avec $\left|\sum_{i=1}^{\bar{k}} \alpha_{i}^{2}(x)-1\right| \leq \tau(\varepsilon)$.

Montrons que $\sum_{i=1}^{\bar{k}}\left|f_{i}(x)-\alpha_{i}(x)\right|^{2}$ est petit.

$$
\sum_{i=1}^{\bar{k}}\left|f_{i}(x)-\alpha_{i}(x)\right|^{2}=\sum_{i=1}^{\bar{k}} f_{i}^{2}(x)+\sum_{i=1}^{\bar{k}} \alpha_{i}^{2}(x)-2 \sum_{i=1}^{\bar{k}} \alpha_{i}(x) f_{i}(x) .
$$

Or le lemme 4.4 implique

$$
\sum_{i=1}^{\bar{k}} f_{i}^{2}(x) \leq 1+\tau(\sqrt{\delta(\varepsilon)}) .
$$

D'autre part, l'inégalité (47) appliquée pour $z=x$ donne

$$
\left|\sum_{i=1}^{\bar{k}} \alpha_{i}(x) f_{i}(x)-1\right| \leq \tau(\varepsilon)
$$

On obtient finalement

$$
\sum_{i=1}^{\bar{k}}\left|f_{i}(x)-\alpha_{i}(x)\right|^{2} \leq 3 \tau(\varepsilon)+\tau(\sqrt{\delta(\varepsilon)}) .
$$

En appliquant l'inégalité (47) à $y$ dans $A_{k}^{R(\varepsilon)}$, il vient

$$
\begin{gathered}
\left|\langle F(x), F(y)\rangle_{\mathbb{R}^{k}}-\cos d(x, y)\right| \leq\left|\sum_{i=1}^{k} f_{i}(x) f_{i}(y)-\sum_{i=1}^{\bar{k}} \alpha_{i}(x) f_{i}(y)\right|+\tau(\varepsilon) \\
\leq\left|\sum_{i=1}^{\bar{k}}\left(f_{i}(x)-\alpha_{i}(x)\right) f_{i}(y)\right|+\left|\sum_{i=k+1}^{\bar{k}} f_{i}(x) f_{i}(y)\right|+\tau(\varepsilon) .
\end{gathered}
$$

En utilisant l'inégalité de Cauchy-Schwartz puis (49) et (48), on obtient

$$
\left|\sum_{i=1}^{\bar{k}}\left(f_{i}(x)-\alpha_{i}(x)\right) f_{i}(y)\right| \leq((3 \tau(\varepsilon)+\tau(\sqrt{\delta(\varepsilon)}))(1+\tau(\sqrt{\delta(\varepsilon)})))^{\frac{1}{2}} .
$$

Il ne reste plus qu'à estimer le terme $\left|\sum_{i=k+1}^{\bar{k}} f_{i}(x) f_{i}(y)\right|$. Or pour tout élément z de $A_{k}^{R(\varepsilon)}$, on a

$$
\sum_{i=1}^{k} f_{i}^{2}(z) \geq 1-R(\varepsilon)
$$


donc en utilisant de nouveau l'inégalité de Cauchy-Schwartz et (48), on obtient

$$
\left|\sum_{i=k+1}^{\bar{k}} f_{i}(x) f_{i}(y)\right| \leq R(\varepsilon)+\tau(\sqrt{\delta(\varepsilon)}),
$$

ce qui termine la démonstration.

Remerciements. Ce travail fait partie de ma thèse de doctorat. Je tiens à remercier chaleureusement mon directeur de thèse, G. Courtois, pour les échanges fructieux et cordiaux initiés à cette occasion.

\section{Références}

[1] Michael T. Anderson, Metrics of positive Ricci curvature with large diameter. Manuscripta Math. 68 (4) (1990), 405-415. Zbl 0711.53036 MR 1068264

[2] Erwann Aubry, Théorème de la sphère. In Séminaire de Théorie Spectrale et Géométrie 18 (Année 1999-2000), Univ. Grenoble I, Saint-Martin-d'Hères 2000, 125-155. Zbl 1078.53518 MR 1812217

[3] Richard L. Bishop and Richard J. Crittenden, Geometry of manifolds. Pure Appl. Math. XV, Academic Press, New York, London 1964. Zbl 0132.16003 MR 169148

[4] Jeff Cheeger and Tobias H. Colding, Lower bounds on Ricci curvature and the almost rigidity of warped products. Ann. of Math. (2) 144 (1) (1996), 189-237. Zbl 0865.53037 MR 1405949

[5] Shiu Yuen Cheng, Eigenvalue comparison theorems and its geometric applications. Math. Z. 143 (3) (1975), 289-297. Zbl 0329.53035 MR 0378001

[6] Tobias H. Colding, Large manifolds with positive Ricci curvature. Invent. Math. 124 (1-3) (1996), 193-214. Zbl 0871.53028 MR 1369415

[7] Tobias H. Colding, Shape of manifolds with positive Ricci curvature. Invent. Math. 124 (1-3) (1996), 175-191. Zbl 0871.53027 MR 1369414

[8] Christopher B. Croke, An eigenvalue pinching theorem. Invent. Math. 68 (2) (1982), 253-256. Zbl 0505.53018 MR 0666162

[9] Sylvestre Gallot, Variétés dont le spectre ressemble à celui de la sphère. Astérisque $\mathbf{8 0}$ (1980), 33-52. Zbl 0471.53027 MR 0620168

[10] Sylvestre Gallot, Inégalités isopérimétriques, courbure de Ricci et invariants géométriques. II. C. R. Acad. Sci. Paris Sér. I Math. 296 (8) (1983), 365-368. Zbl 0535.53035 MR 0699164

[11] Sylvestre Gallot, Volumes, courbure de Ricci et convergence des variétés (d'après T. H. Colding et Cheeger-Colding). Séminaire Bourbaki 1997/98; Astérisque 252 (1998), 7-32. Zbl 0976.53038 MR 1685585

[12] Karsten Grove and Peter Petersen, V., A pinching theorem for homotopy spheres. J. Amer. Math. Soc. 3 (3) (1990), 671-677. Zbl 0717.53025 MR 1049696 
[13] Peter Li and Shing Tung Yau, Estimates of eigenvalues of a compact Riemannian manifold. In Geometry of the Laplace operator (Honolulu, Hawaii, 1979), Proc. Sympos. Pure Math. XXXVI, Amer. Math. Soc., Providence, R.I., 1980, 205-239. Zbl 0441.58014 MR 0573435

[14] André Lichnerowicz, Géométrie des groupes de transformations. Travaux et Recherches Mathématiques III, Dunod, Paris 1958. Zbl 0096.16001 MR 0124009

[15] Sumner B. Myers, Riemannian manifolds with positive mean curvature. Duke Math. J. 8 (1941), 401-404. Zbl 0025.22704 MR 0004518

[16] Morio Obata, Certain conditions for a Riemannian manifold to be iosometric with a sphere. J. Math. Soc. Japan 14 (1962), 333-340. Zbl 0115.39302 MR 0142086

[17] Peter Petersen, On eigenvalue pinching in positive Ricci curvature. Invent. Math. 138 (1) (1999), 1-21. Zbl 0988.53011 MR 1714334

[18] Peter Petersen, Erratum : "On eigenvalue pinching in positive Ricci curvature" Invent. Math. 155 (1) (2004), 223. MR 2025305

[19] Richard Schoen and Shing-Tung Yau, Lectures on differential geometry. Conf. Proc. Lecture Notes Geom. Topology I, International Press, Cambridge, MA, 1994. Zbl 0830.53001 MR 1333601

Received June 29, 2004; revised November 28, 2005

Jérôme Bertrand, Institut de Mathématiques, Université P. Sabatier, 118 route de Narbonne, 31000 Toulouse Cedex 4, France

E-mail: bertrand@math.ups-tlse.fr 